\title{
Effect of Varying Frequency of a Synthetic Jet on Flow Separation Over an Airfoil
}

\author{
M. Kim, E.E. Essel, P.E. Sullivan \\ Version Accepted Manuscript \\ Citation M. Kim, E.E. Essel, and P.E. Sullivan, "Effect of varying frequency of a \\ (published version) synthetic jet on flow separation over an airfoil", Phys. Fluids 34, 015122 \\ (2022). https://doi.org/10.1063/5.0077334 \\ Publisher's Statement This article may be downloaded for personal use only. Any other use \\ requires prior permission of the author and AIP Publishing. This article \\ appeared in Physics of Fluids, Volume 34, Issue 1, and may be found at \\ https://doi.org/10.1063/5.0077334.
}

\section{How to cite TSpace items}

Always cite the published version, so the author(s) will receive recognition through services that track citation counts, e.g. Scopus. If you need to cite the page number of the author manuscript from TSpace because you cannot access the published version, then cite the TSpace version in addition to the published version using the permanent URI (handle) found on the record page.

This article was made openly accessible by $U$ of T Faculty. Please tell us how this access benefits you. Your story matters. 


\author{
M. Kim, E.E. Essel, P.E. Sullivan \\ Turbulence Research Lab \\ Dept of Mechanical and Industrial Engineering \\ University of Toronto \\ Toronto, ON M5S 3 G8 Canada
}

\section{ABSTRACT}

An experimental investigation on the effects of the synthetic jet actuator (SJA) was conducted on a NACA (National Advisory Committee for Aeronautics) 0025 airfoil in a low-speed recirculating wind tunnel at a chord Reynolds number of 100,000 and at an angle of attack $12^{\circ}$. Particle image velocimetry was used to visualize the flow separation for the uncontrolled baseline flow, and the flow attachment for the SJA controlled flows. The location of the SJA was at $-1.3 \%$ from the separation point, and a blowing ratio of 0.8 was chosen for this study. The blowing ratio proved to be effective in suppressing the separation of the flow. The reduced frequency $\left(S t_{e}\right)$ was varied between $1,2,14$, and 58 . The momentum bursts from the SJA based on the reduced frequency determined the effectiveness of the control method. The Reynolds stresses and turbulence production decreased dramatically with increasing frequency up to the shear layer frequency $\left(S t_{e}=14\right)$, but further excitation $\left(S t_{e}=58\right)$ resulted in a regain of turbulence levels. Proper orthogonal decomposition (POD) was performed which showed that the low frequency operations globally affect the modes in the shear layer while the high frequency operations are confined to the airfoil surface.

\section{NOMENCLATURE}

$A \quad$ matrix of $u^{\prime}$ and $v^{\prime}$ vectors in each column for each timestep for the full timesteps

$a_{i}(t) \quad$ coefficients corresponding to the $\mathrm{i}^{\text {th }}$ mode

$\alpha \quad$ angle of attack

c airfoil chord

C matrix consisting of temporal coefficients

$C_{B} \quad$ blowing ratio

$C_{m} \quad$ momentum coefficient

$C_{\mu} \quad$ standard modelling coefficient for eddy viscosity models

$d \quad$ orifice diameter or slot width

$f_{e} \quad$ excitation frequency

$F^{+} \quad$ reduced frequency (based on distance from the slot exit to the trailing edge) 


\begin{tabular}{rll}
1 & $\mathrm{i}$ & $\mathrm{i}^{\text {th }}$ mode \\
2 & $k$ & turbulent kinetic energy \\
3 & $L_{v}$ & vortex formation length \\
4 & $\mathrm{M}$ & number of timesteps \\
5 & $\mathrm{~N}$ & number of data points in the velocity vector field for $\mathrm{u}^{\prime}$ and $\mathrm{v}$ \\
6 & $\phi_{i}(x)$ & eigenfunction of the $\mathrm{i}^{\text {th }}$ mode \\
7 & $\phi_{\mathrm{u}}$ & eigenfunctions representing the streamwise velocity \\
8 & $\phi_{\mathrm{v}}$ & eigenfunctions representing the transverse velocity \\
9 & $P_{N}$ & contribution of normal stresses in the production of turbulent kinetic energy \\
10 & $P_{s}$ & contribution of shear stresses in the production of turbulent kinetic energy \\
11 & $P_{t k e}$ & production of turbulent kinetic energy \\
12 & $R e_{c}$ & chord Reynolds number \\
13 & $\sum$ & eigenvalue matrix from the proper orthogonal decomposition \\
14 & $S t_{e}$ & excitation Strouhal number, reduced frequency (based on chord length) \\
15 & $\overline{u^{\prime} u^{\prime}}$ & streamwise Reynolds normal stress \\
16 & $\overline{u^{\prime} v^{\prime}}$ & Reynolds shear stress \\
17 & $U$ & streamwise mean velocity \\
18 & $U_{c}$ & convection velocity \\
19 & $U_{\infty}$ & freestream velocity \\
20 & $\bar{U}_{J}$ & phase averaged jet velocity \\
21 & $U_{m a x}$ & local streamwise maximum velocity \\
22 & $\overline{v^{\prime} v^{\prime}}$ & transverse Reynolds normal stresses \\
23 & $\mathrm{~V}$ & transverse mean velocity \\
24 & $\mathrm{~W}$ & matrix consisting of spatially orthogonal modes \\
25 & $x$ & chordwise coordinate originating at the leading edge \\
26 & $x_{j}$ & location of jet \\
27 & $\mathrm{X}$ & global streamwise coordinate \\
28 & $X_{t e}$ & distance from the slot to the trailing edge \\
29 & $y$ & coordinate normal to the airfoil surface \\
30 & $\mathrm{Y}$ & global transverse coordinate \\
31 & $\mathrm{Z}$ & global spanwise coordinate \\
& & \\
\hline
\end{tabular}

\section{I. INTRODUCTION}

33 Low Reynolds number flows around airfoils are prevalent in diverse engineering applications including 34 propellers, sailplanes, unmanned aerial vehicles (UAV), wind turbines and low-speed/high altitude aircraft.

35 These low Reynolds number operations, defined based on the airfoil chord Reynolds number, $\operatorname{Re}_{c}=10^{5}-$

$365 \times 10^{5}$, differ significantly from high Reynolds number flows such as conventional, large scale, high speed 37 aircraft with typical $R e_{c}=10^{6}-10^{9} \cdot{ }^{1-3}$ In particular, at low Reynolds number, there is a rapid growth of 38 a laminar boundary layer (LBL) on the upper surface of the airfoil. The LBL often separates near the leading 39 edge of the airfoil due to an adverse pressure gradient (APG) to form a separated shear layer that undergoes 40 laminar-to-turbulent transition. For airfoils operated at relatively low angle of attack $(\alpha)$, the separated shear 
1 layer may reattach on the airfoil surface to form the well-known laminar separation bubble (LSB). However,

2 at high angle of attack, the separated shear layer fails to reattach forming a wide wake of stall or post-stall

3 flow. The flow separation and occurrence of stall has detrimental effects on aerodynamic performance by

4 decreasing lift and increasing drag of the airfoil. ${ }^{2,4}$ Moreover, the unsteadiness of the separated shear layer

5 and the wake may lead to vortex-induced vibrations, noise generation, structural loads and fatigue failure.

6 Given these undesirable effects, flow control for low Reynolds number airfoils continues to be an active

7 research area with the motivation of understanding the complex flow structure and developing effective

8 control strategies for improving aerodynamic performance.

9 Over the past few decades, considerable effort has been devoted to drag reduction of airfoils using various passive and active control strategies. ${ }^{5-8}$ Passive control can include vortex generators such as trip wires and surface roughness to delay flow separation while active control uses steady or periodically excited devices

12 (e.g., actuators) that can be operated to achieve desired outcomes. ${ }^{9}$ In many applications, active control 13 devices are preferred because of their flexibility in off-design conditions and they do not introduce a drag 14 penalty common with passive control devices permanently mounted on the airfoil. Among the active control strategies, the synthetic jet (also known as zero-net-mass flux or ZNMF jet) has shown promise as it possesses the features of both the steady blowing or suction devices and the pulsed jet. ${ }^{4,8,10-13}$ It has proven to be more energy-efficient than the conventional steady blowing or suction devices since it requires one to two orders of magnitude less momentum to produce similar control effects. ${ }^{9}{ }^{14}$ The synthetic jet actuator (SJA) consists of a vibrating diaphragm mounted in a cavity with the exit (i.e., orifice or slot) located on the surface of the airfoil. Periodic excitation is applied to the diaphragm which causes the working fluid to be alternatively ingested and expelled by the cavity, thereby locally transferring linear momentum and zeronet mass into the flow. ${ }^{15}$ The net momentum added to the flow during the expulsion phase is attributed to the formation of vortex pairs at the orifice or slot exit(s). ${ }^{10,16}$ The use of the working fluid for flow control is the unique feature of the SJA that differentiates it from the pulsed jet.

Previous studies on the application of the SJA to enhance airfoil performance have shown that the effectiveness of the SJA depends on important parameters such as the reduced frequency $\left(F^{+}=f_{e} X_{t e} / U_{\infty}\right)$, ${ }^{11,17-23}$ blowing ratio $\left(C_{B}=\bar{U}_{J} / U_{\infty}\right)^{11,22}$ or momentum coefficient $\left(C_{m}=\bar{U}_{J}{ }^{2} d /\left(0.5 U_{\infty}{ }^{2} c\right)\right),{ }^{10,24}$ and synthetic jet location ${ }^{11,17}$, where $f_{e}$ is the excitation frequency, $X_{t e}$ is the distance from the slot to the trailing edge of the airfoil, $c$ is the chord length, $U_{\infty}$ is the freestream velocity, $\bar{U}_{J}$ is the phase-averaged jet velocity and $d$ is the orifice diameter or slot width. A summary of relevant studies and the geometric and flow conditions investigated are presented in Table I. Comprehensive reviews of previous investigations can also be found in Greenblatt \& Wygnanski ${ }^{9}$ and Zhang \& Samtaney ${ }^{25}$. Greenblatt \& Wygnanski ${ }^{9}$ summarized the results of previous studies and found that the optimum reduced frequency to cause attachment of the 
1 separated shear layer is within the range of $0.3 \leq F^{+} \leq 4.0$. This range of reduced frequency corresponds 2 to an excitation Strouhal number, $S t_{e}=f_{e} c / U_{\infty}$ of $\boldsymbol{O}(1)$. Goodfellow et al. ${ }^{10}$ investigated the effects of 3 momentum coefficient on boundary layer separation and reattachment and found that applying a periodic 4 excitation above the threshold $C_{m}$ level causes flow attachment, significant reduction in wake width and 5 about $50 \%$ reduction in drag. In terms of the synthetic jet location, Amitay et al. ${ }^{19}$ proposed that the slot of 6 the actuator should be placed near the separation point of the baseline condition for angle of attacks, $\alpha \in$ $7\left[5^{\circ}, 25^{\circ}\right]$. Although the results of Seifert \& Pack $^{26}$ agreed with the findings of Amitay et al. ${ }^{19}$, Raju et al..$^{27}$ 8 and Kotapati et $a l .{ }^{28}$ argued that positioning the synthetic jet exit near the separation point may not improve the lift-to-drag ratio. Buchmann et al. ${ }^{29}$ also showed that the synthetic jet positioned at the leading edge of

11 Feero et al. ${ }^{11}$ conducted a recent experimental investigation on the effect of synthetic jet slot location using 12 a NACA 0025 airfoil at $R e_{c}=1.0 \times 10^{5}$ and $\alpha=12^{\circ}$. Four slot locations measured relative to the 13 separation point of the baseline flow were tested with two locations upstream $\left(x_{j} / c=-4.3 \%\right.$ and $\left.-1.3 \%\right)$ 14 and the other two downstream $\left(x_{j} / c=1.3 \%\right.$ and $\left.4.3 \%\right)$ of the separation point. They measured the drag and lift coefficients for each slot location and for varying reduced frequencies and blowing ratios and found that the lift-to-drag ratio increased as the slot location moved upstream for both low- and high- reduced frequencies.

Two dominant features are generally associated with the post-stall separated flow over an airfoil: (a) vortex roll-up of the separated shear layer and (b) vortex shedding of large-scale structures in the wake. Studies have found the vortex shedding of a post-stall airfoil to have similar characteristics as the wake of a bluff body. ${ }^{18,30}$ While multiple studies have focused on the vortex shedding frequency, ${ }^{10,20,21,31}$ Yarusevych et al. ${ }^{32,33}$ found the vortex roll up to be associated with the fundamental frequency of the shear layer, while vortex merging was related to the subharmonic growth and transition to turbulence. Studies on the relationship between shear layer instability and wake instability have showed nonlinear coupling between the two instabilities, ${ }^{34}$ and have established the ratio between the shear layer frequency and vortex shedding frequency to be proportional to the power of the Reynolds number of the flow. ${ }^{35-37}$ For high frequency actuation, Amitay \& Glezer ${ }^{38}$ found the actuation frequency of $\boldsymbol{O}(10)$ to be effective as it alters the aerodynamics and creates a favorable pressure gradient.

The objective of this study is to characterize the effects of varying frequency and momentum of an SJA on flow separation over a NACA 0025 airfoil using a high-resolution particle image velocimetry (PIV) system. The experiments were conducted at a fixed Reynolds number of $R e_{c}=1.0 \times 10^{5}$, but the excitation Strouhal number was varied at $S t_{e}=1,2,14$ and 58 to induce flow control at various dominant shear layer and wake frequencies. While most previous studies focused on the influence of the SJA on the aerodynamic 
1 performance of the airfoil using drag and lift measurements, the present study provides additional insights 2 on the impact on the Reynolds stresses and their production, and the dynamics of the large-scale structures 3 using proper orthogonal decomposition (POD) analysis. 
TABLE 1 Summary of relevant experiments or numerical studies using synthetic jet actuator on flow control around airfoil.

\begin{tabular}{|c|c|c|c|c|c|}
\hline Authors & Airfoil & Technique & Angle of Attack $(\alpha)$ & $R e_{C}\left(U_{\infty} c / v\right) \times 10^{5}$ & $S t_{e}\left(f c / U_{\infty}\right)$ \\
\hline Hsiao et al. ${ }^{17}$ & NACA $63_{3-018}$ & $\begin{array}{l}\text { Smoke wire, pressure } \\
\text { taps, Hot-wire }\end{array}$ & $0-26$ & $0.063-5.00$ & $0-20.0^{*}$ \\
\hline Wu et al. ${ }^{18}$ & NACA 0012 & LES & $18-35$ & 5.00 & $0-40.0^{*}$ \\
\hline Seifert \& Pack ${ }^{26}$ & $\begin{array}{l}\text { NACA 0012/ } \\
\text { NACA 0015 }\end{array}$ & Pressure taps & $6-14$ & $15.00-23.5$ & $\sim 2.2^{*}$ \\
\hline Amitay et al. ${ }^{19,20}$ & NACA & Pressure taps, PIV & $\begin{array}{l}0,5,10,15,17.5 \\
20,25\end{array}$ & $3.10-7.25$ & $1.0-20.0$ \\
\hline Gilarranz, J. L. et al. ${ }^{21}$ & NACA 0015 & $\begin{array}{l}\text { Pressure taps, } \\
\text { oil, tuft, smoke, } \\
\text { visualization }\end{array}$ & $2-29$ & 8.96 & $0.6-1.4^{*}$ \\
\hline Duvigneau, R. \& Visonneau, M. ${ }^{31}$ & NACA 0015 & URANS & $12-24$ & 8.96 & 1.3 \\
\hline Raju, R. et al. ${ }^{27}$ & NACA 4418 & IBM & 18 & 0.4 & $1.0-12.0$ \\
\hline You, D. \& Moin, P. ${ }^{23}$ & NACA 0015 & LES & $12-18$ & 8.96 & $0.7-1.4$ \\
\hline Buchmann, N. A. et al. ${ }^{29}$ & NACA 0015 & PIV & 18 & 0.3 & 1.3 \\
\hline Goodfellow S. D. et al. ${ }^{10}$ & NACA 0025 & Smoke wire & 5 & 1.00 & $28.8-51.9^{*}$ \\
\hline Zhang, W. \& Samtaney, R. ${ }^{25}$ & NACA 0018 & DNS & 10 & 0.1 & $0.5,1.0,4.0$ \\
\hline Montazer, E et al. ${ }^{22}$ & NACA 0015 & RANS & 13,16 & 8.96 & $\sim 1.1^{*}$ \\
\hline Moshfeghi, M. \& Hur, N. ${ }^{24}$ & S809 & DES & $0-25$ & 10 & $1.3^{*}$ \\
\hline Feero, M. A. et al. ${ }^{11}$ & NACA 0025 & PIV & 12 & 1.00 & $\begin{array}{l}1.0,2.0,14.0, \\
58.0\end{array}$ \\
\hline
\end{tabular}

LES: Large eddy simulation, URANS: Unsteady Reynolds averaged Navier Stokes, IBM: Immersed boundary method, DNS: Direct numerical simulation, RANS: Reynolds average Navier Stokes, DES: Detached eddy simulation

* Values of the reduced frequency were calculated based on the information provided in the paper 


\section{EXPERIMENTAL CONDITIONS}

2 The experiments were conducted on a NACA 0025 airfoil in the recirculating wind tunnel facility at the

3 University of Toronto. The test section of the wind tunnel is $5 \mathrm{~m}$ long with a width and height of $0.91 \mathrm{~m}$ and $1.22 \mathrm{~m}$, respectively. Fig. 1 shows a schematic of the experimental setup. The experiments were conducted at a freestream velocity of $U_{\infty}=5.2 \mathrm{~m} / \mathrm{s}$ and turbulence intensity of $0.08 \%$. The airfoil has a chord length of $c=300 \mathrm{~mm}$, with spanwise length of $885 \mathrm{~mm}$ and a resulting aspect ratio of about 3 . The Reynolds number based on the freestream velocity and chord length, $R e_{c}=1.0 \times 10^{5}$. The airfoil was positioned $1.33 \mathrm{c}$ from the inlet of the test section and fitted with circular acrylic end plates at each end of the model to suppress end effects and the influence of the tunnel sidewall boundary layer. The acrylic end plates also allowed for optical access to the cameras. A rotation lock and bearing housings were attached to the end plates to position the airfoil to the desired angle of attack. For this work, the angle of attack was set to $12^{\circ}$ for each experiment to achieve a fully separated flow on the suction side of the baseline flow condition. As shown in Fig. 1(b), the global coordinate system adopted have the origin fixed at $0.25 c$ from the leading edge of the airfoil and these coordinates are denoted as $[\mathrm{X}, \mathrm{Y}, \mathrm{Z}]$ for the streamwise, transverse and spanwise directions, respectively.
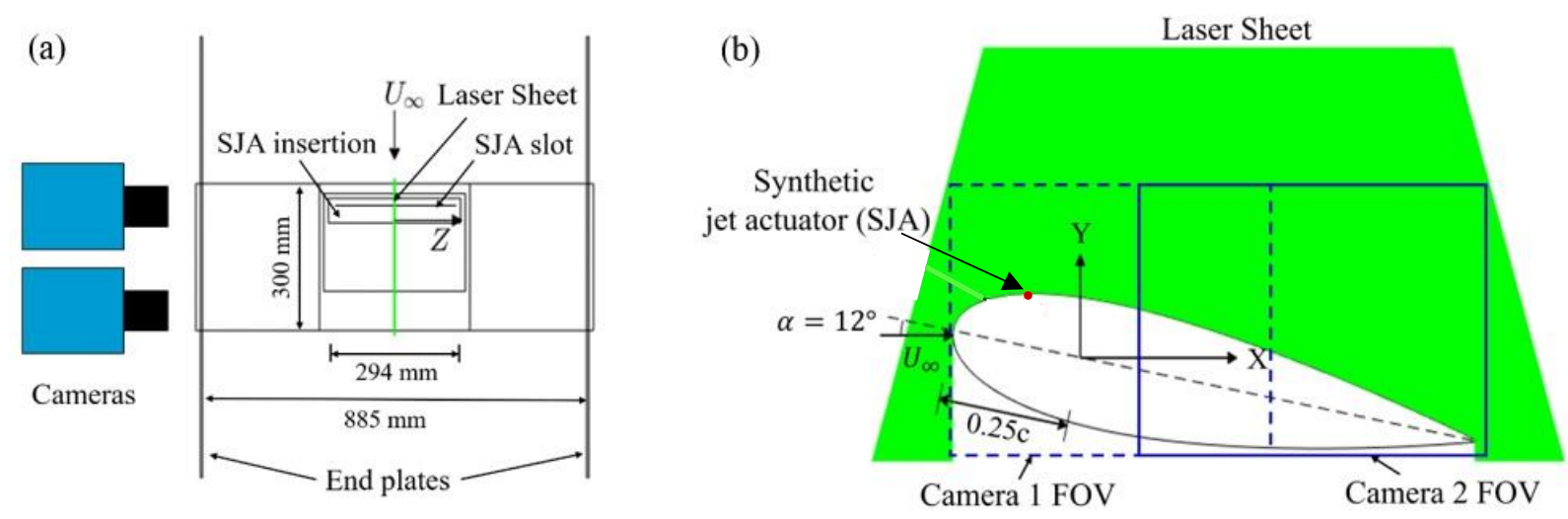

FIG. 1. (a) Schematic of a NACA0025 airfoil and (b) layout of the experimental setup.

The SJA was mounted inside the airfoil with exit on the surface to provide uniform forcing over approximately $1 / 3$ of the model span (Fig. 1(a)). The SJA has a single slot that is $0.5 \mathrm{~mm}$ wide and $294 \mathrm{~mm}$ long in which flow was ejected normal to the airfoil surface. Based on previous studies ${ }^{11}$, the exit slot of the SJA was positioned $0.117 c$ from the leading edge, corresponding to $x_{j} / c=-1.3 \%$ from the separation point of the baseline flow for flow control. The slot is connected to a single cavity driven by 16 piezoelectric diaphragms each of diameter $35 \mathrm{~mm}$. The diaphragms are installed on the two opposite walls of the cavity that are parallel to the slot with eight diaphragms per wall. These diaphragms were operated by a function generator connected to a Mide QPA3202 50:1 voltage amplifier and it was ensured that they were in-phase 
1 within $\pm 5^{\circ}$ based on velocity measurements across the slot length. Detailed description of the SJA and the 2 characteristics of the jet velocity profiles are available in Feero ${ }^{39}$. Here, the blowing ratio was set to $C_{B}=$ 30.8 , to ensure flow attachment for all the frequencies investigated. Four reduced forcing frequency were 4 tested, $S t_{e}=1,2,14$ and 58, denoted as F1, F2, F14 and F58, respectively and a reference baseline flow. 5 Feero et al. ${ }^{11}$ noted the reduced frequency of the vortex shedding frequency was 1 and the reduced frequency 6 of the shear layer instability was $18 \pm 8$. Therefore, the forcing frequencies of the SJA were chosen such that 7 the frequency of the SJA matched that of the vortex shedding frequency (F1) and the frequency of the shear 8 layer instability (F14). F2 was chosen as the first harmonic of the vortex shedding frequency, and F58 was 9 chosen to study a high frequency strategy. For F1, F2 and F14, the frequencies were obtained by burst modulation of the carrier high frequency excitation of F58. A burst-modulated waveform consists of a sine wave at a carrier frequency, $f_{c}$ modulated by a square wave at the modulation frequency, $f_{m}$ with duty cycle, where $f_{m}<f_{c}$. The square wave varies between 0 and 1 with the sine wave occurring in "bursts".

PIV was used to measure the whole velocity field in the streamwise-transverse plane at the midspan $(\mathrm{Z}=0)$ of the airfoil. A Quantel EverGreen dual pulsed Nd-YAG $532 \mathrm{~nm}$ laser with a pulse energy of $200 \mathrm{~mJ}$ and a repetition rate of $15 \mathrm{~Hz}$ was used to illuminate plane of interest through a series of optical lenses. A LaVision aerosol generator was used to seed the flow with $1.7 \mu \mathrm{m}$ sebecate droplets with specific gravity of 0.91 . The flow field was captured using two LaVision sCMOS cameras each with a resolution of $2560 \times 2160$ pixels. The size of each field of view (FOV) was $218 \mathrm{~mm} \times 184 \mathrm{~mm}$ with an overlap of $40 \%$ (Fig. 1(b)). Nikon $60 \mathrm{~mm}$ lenses were mounted on the cameras with a minimum f-stop of 2.8. A sample size of 500 image pairs were acquired at a frequency of $5 \mathrm{~Hz}$. The images were processed using the LaVision DaVis software, where the velocity vectors were calculated using a sequential cross correlation algorithm that had a single pass window of $32 \times 32$ interrogation area, followed by two passes with $16 \times 16$ window area with a $50 \%$ overlap. The resulting spatial resolution was $0.0023 c$ and the velocity vectors in the $\mathrm{X}$ and Y directions were $490 \times 265$, respectively.

The uncertainty quantification of PIV measurements depends on the bias and precision errors. The bias errors include inaccurate estimation of the time between laser pulses, optical distortion of the lens, error in the camera's magnification factor, and inaccurate response time of the tracer particles. In this study, the bias errors were significantly reduced by carefully following the PIV precautionary measures outlined by Prasad et al. ${ }^{40}$ and Forliti et al. ${ }^{41}$ and using advanced evaluation algorithms. On the other hand, the precision error corresponds to computational errors of the time-averaged velocities and turbulence statistics. The precision error largely depends on the sample size as these errors can be averaged out by using large 
1 estimated based on the procedure outlined in Sciacchitano \& Wieneke ${ }^{42}$. The uncertainty in the mean

2 velocities and Reynolds stresses at $95 \%$ confidence level $\left(Z_{c}=1.96\right)$ is $\pm 3 \%$ and $\pm 12 \%$, respectively.

\section{III. RESULTS AND DISCUSSIONS}

\section{A. Mean flow}

5 Contours of the streamwise mean velocity for the baseline and the controlled test cases (F1, F14 and F58)

6 are presented in Fig. 2(a-d). The time-averaged streamlines are superimposed on the contours to reveal the

7 mean flow pattern and the contour line for $0.99 U_{\infty}$ is used to differentiate the freestream from the separated

8 shear layer or attached flow. The corresponding plot for F2 is like F1, and therefore, not shown for brevity.

9 The baseline flow (Fig. 1(a)) exhibits a large recirculation region that starts near the leading edge $(\mathrm{X} / \mathrm{c}=$ -

10 0.12) and extends into the wake behind the airfoil, indicating a post-stall flow. The focus of the recirculation

11 region is near the trailing edge $(\mathrm{X} / \mathrm{c}=0.78, \mathrm{Y} / \mathrm{c}=0.02)$. For the controlled flows, the actuation of the

12 synthetic jet suppressed flow separation and delayed the start location $(\mathrm{X} / \mathrm{c} \approx 0.20)$ of the attached flow on

13 the airfoil surface. However, the forcing frequencies associated with the wake instability (F1 and F2) result

14 in a fully attached flow (Fig. 1(b)), while actuation at the shear layer frequency (F14) and F58 result in an

15 attached flow with the onset of a mild separation near the trailing edge of the airfoil. The mild separation

16 is attributed to the build up of adverse pressure gradient near the trailing edge, which is more severe for

17 F58 than F14. This suggests that the use of higher frequency excitation without any correlation to the shear

18 layer instability is an ineffective strategy for separation control. The present results also provide further

19 insight into the drag measurements by Feero et al. ${ }^{11}$ which showed that the drag decreased as the forcing

20 frequency increased from F1 to F14, but further increase to F58 caused an increase in drag. 
(a)

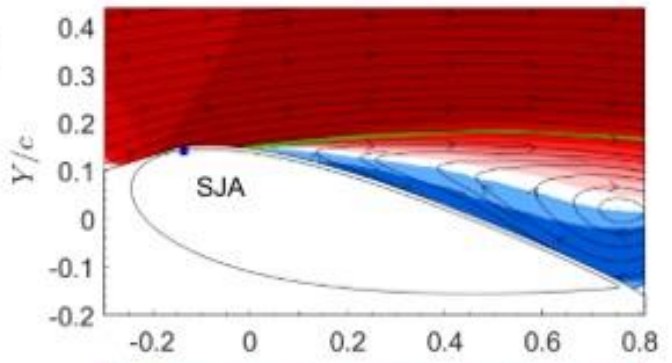

(b)

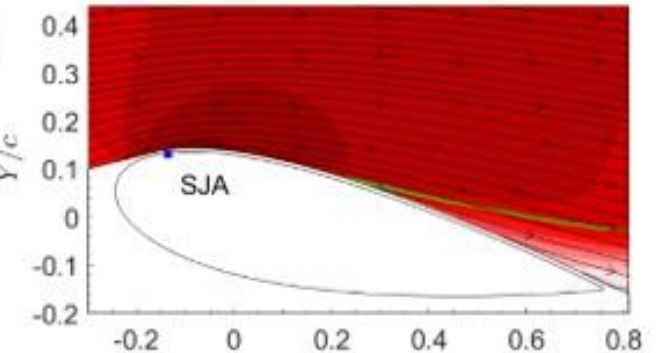

(c)

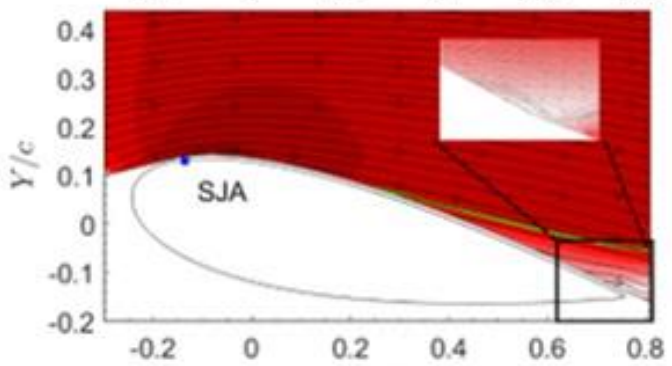

(d)

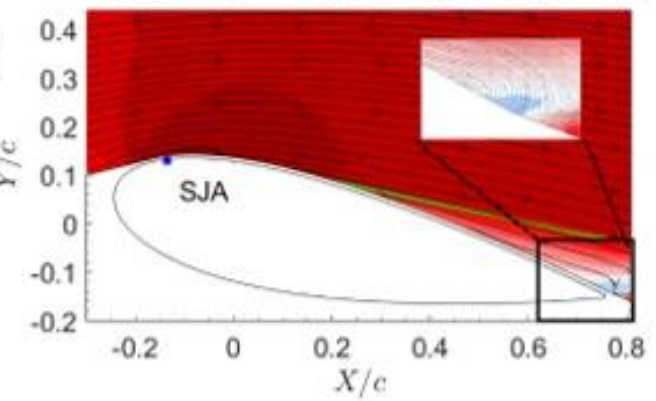

(e)

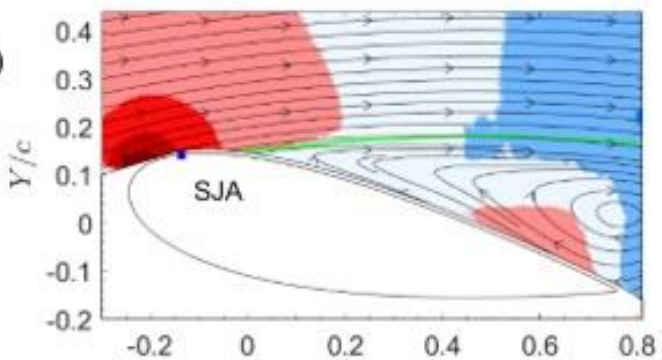

(f)

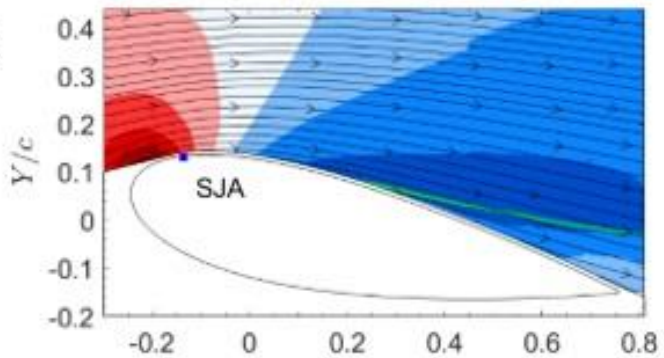

(g)

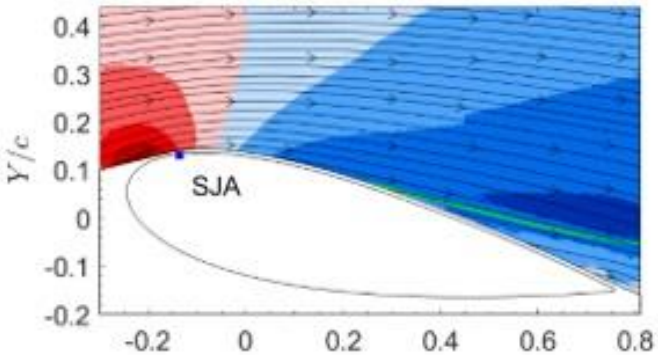

(h)

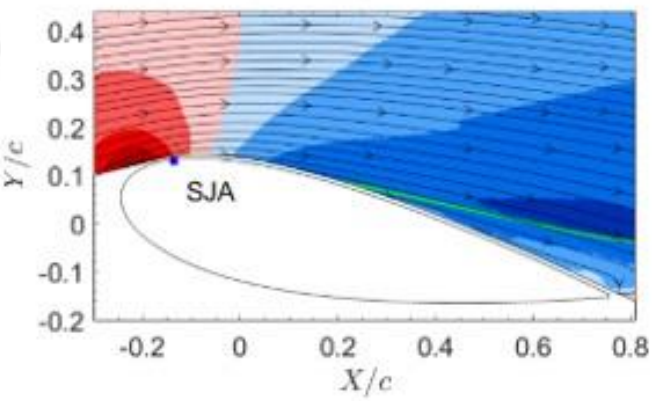

FIG. 2. Contours of streamwise and transverse mean velocities for Baseline (a, e), F1 (b, f), F14 (c, g) and F58 $(\mathrm{d}, \mathrm{h})$. Inset shows magnification of the bottom right corner where a minor separation and reattachment in shown.

2 The effects of the forcing frequency on the streamwise evolution of the bulk flow over the airfoil is examined using the distributions of the normalized local maximum mean velocities $\left(U_{\max } / U_{\infty}\right)$ shown in Fig. 3. For each test case, the approach flow rapidly increases at the leading edge of the airfoil to a local maximum at $\mathrm{X} / c \approx-0.2$ for the baseline flow and $\mathrm{X} / c \approx-0.1$ for the controlled cases. The additional momentum input by the SJA contributes to about $14 \%$ increase in the peak velocity for the controlled flow, but this peak value is independent of forcing frequency. Further downstream, the local peak for the baseline 
flow rapidly decreases and plateau over the rest of the airfoil. It is interesting to notice that, the streamwise

2 location where the plateau starts also coincides with the mean separation point of the baseline flow. In the

3 case of the SJA flows, the profiles gradually decrease beyond the peak location and asymptote at the onset

4 of the attached flow region $(\mathrm{X} / c \geq 0.2)$. The local maximum velocity in the attached flow region is about

$57 \%-14 \%$ lower than the plateau values of the baseline flow. This momentum deficit for the controlled

6 cases is caused by the strong entrainment of freestream fluid towards the airfoil surface (Fig. 2(f)-(h)) to

7 counteract the adverse pressure gradient near the surface. The transition region $(X / c \in[-0.1,0.2])$ in the

8 profiles of the controlled cases is often characterized by temporal formation of LSBs and their subsequent

9 breakdown due to the opposing effects of vortex structures generated by the SJA ${ }^{11,29}$.

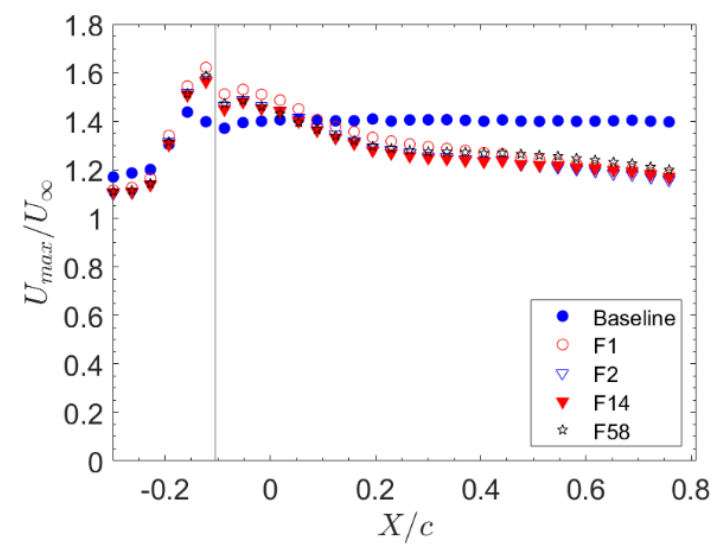

FIG. 3. Distribution of the normalized maximum mean streamwise velocity. The line shows the flow separation point for the baseline flow.

\section{B. Reynolds stresses}

Figures 4 and 5 compare the contours of the Reynolds normal $\left(\overline{u^{\prime} u^{\prime}}\right.$ and $\left.\overline{v^{\prime} v^{\prime}}\right)$ and cross $\left(-\overline{u^{\prime} v^{\prime}}\right)$ stresses for the baseline flow and the controlled cases. The Reynolds stresses are normalized by the freestream velocity. For the baseline flow, the flow is laminar prior to the separation point, therefore, the Reynolds stresses are negligible in that region. After the separation point, the Reynolds stresses significantly increases in the separated shear layer of the baseline flow due the Kelvin Helmholtz shear layer instability and the associated quasi-periodic vortex shedding. Unlike $\overline{v^{\prime} v^{\prime}}$, the intense region of $\overline{u^{\prime} u^{\prime}}$ and $-\overline{u^{\prime} v^{\prime}}$ persist further downstream due to the strong mean shear $(\partial U / \partial y)$ in the stalled flow. On the other hand, the Reynolds stresses for the controlled flows are substantially reduced and shifted closer to the airfoil surface compared to the baseline flow. The reduced Reynolds stresses is an artifact of the separation control by the SJA, and the associated suppression of the shear layer instability observed in the baseline flow. Therefore, most of the Reynolds stresses in the controlled cases are generated from the large mean shear near the attached flow surface and the separation at the trailing edge of the airfoil. A significant improvement in lift and reduction 
1 in drag can be obtained by the SJA as the reduction of the Reynolds stresses decreases energy losses from

2 the mean flow and drag. For the various forcing frequencies, F1 shows Reynolds stresses that extended out

3 of the attached flow region, while F14 shows the thinnest region of high Reynolds stresses. While the

4 Reynolds stresses decrease as actuation frequencies increases from F1 to F14, F58 shows an increase in

5 Reynolds stresses compared to F14.
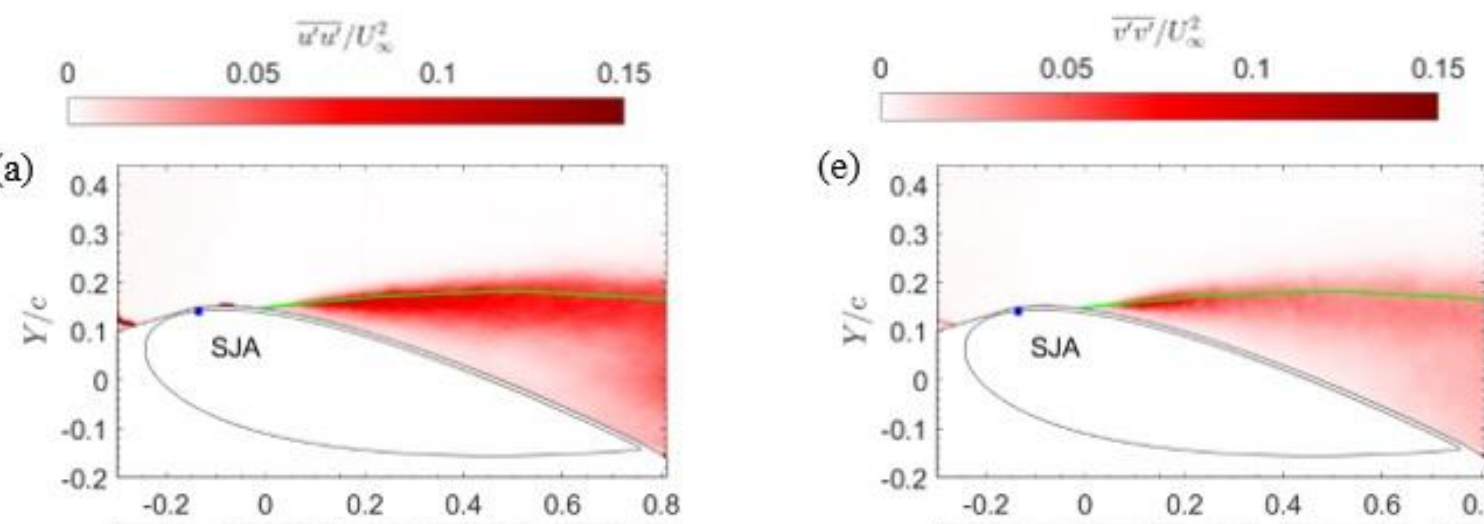

(a)

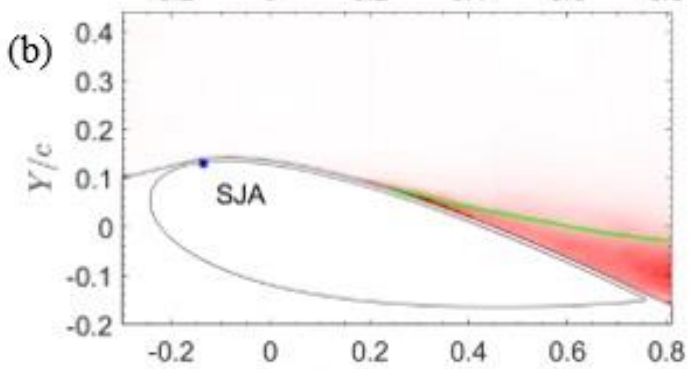

(c)
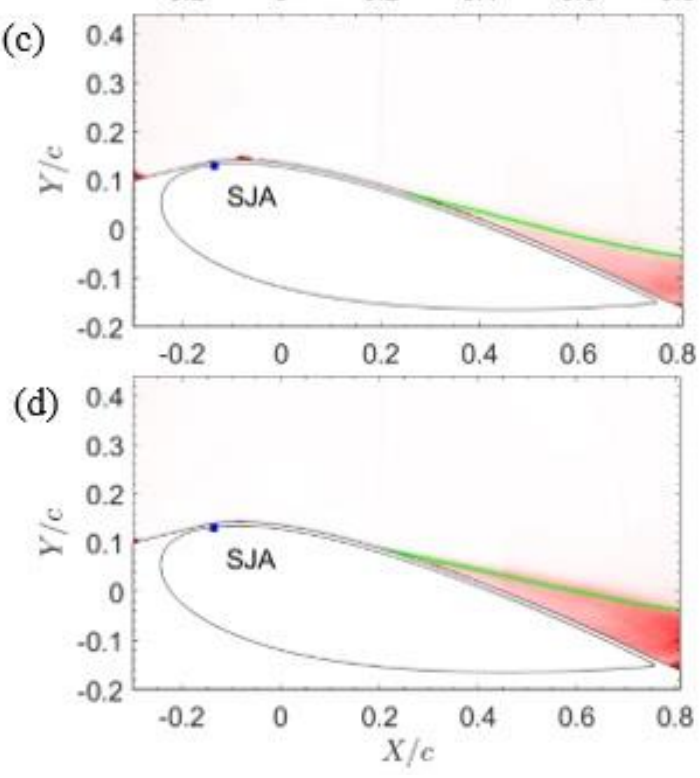

(e)

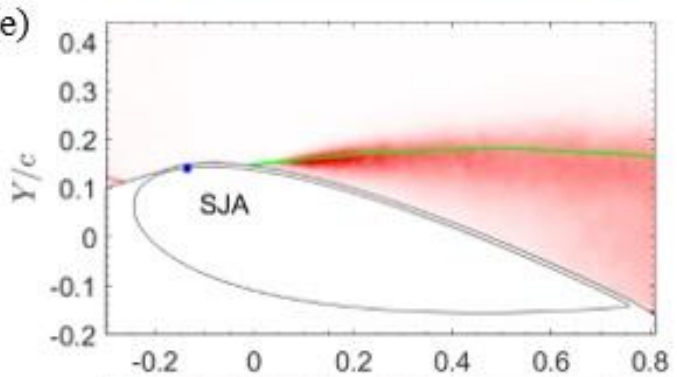

(f)

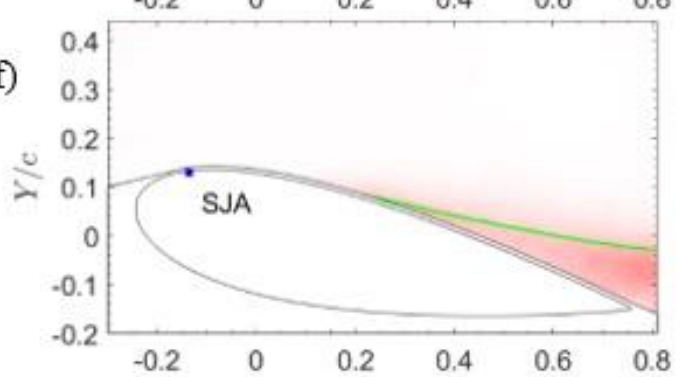

(g)

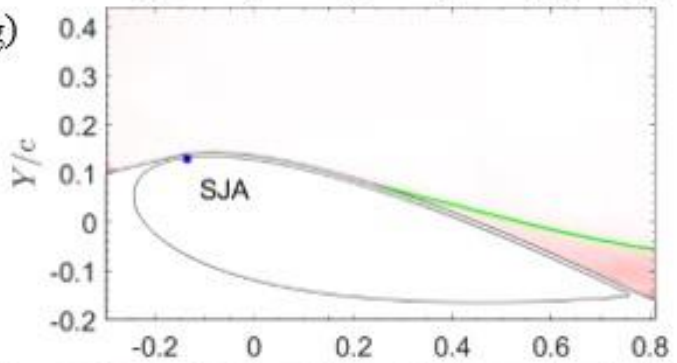

(h)

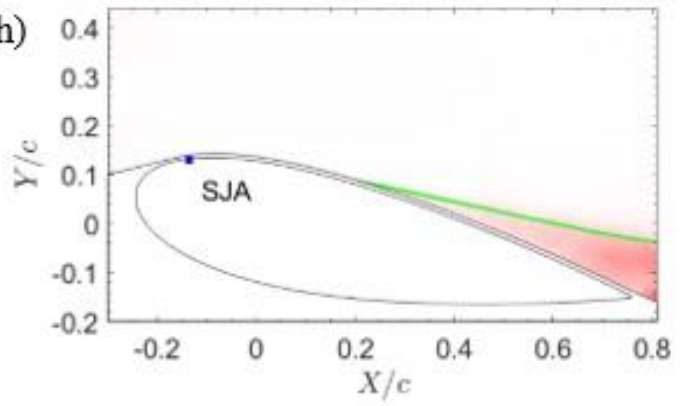

FIG. 4. Contours of streamwise and spanwise Reynolds normal stresses for Baseline (a, e), F1 (b, f), F14 $(\mathrm{c}, \mathrm{g})$ and F58 (d, h). 

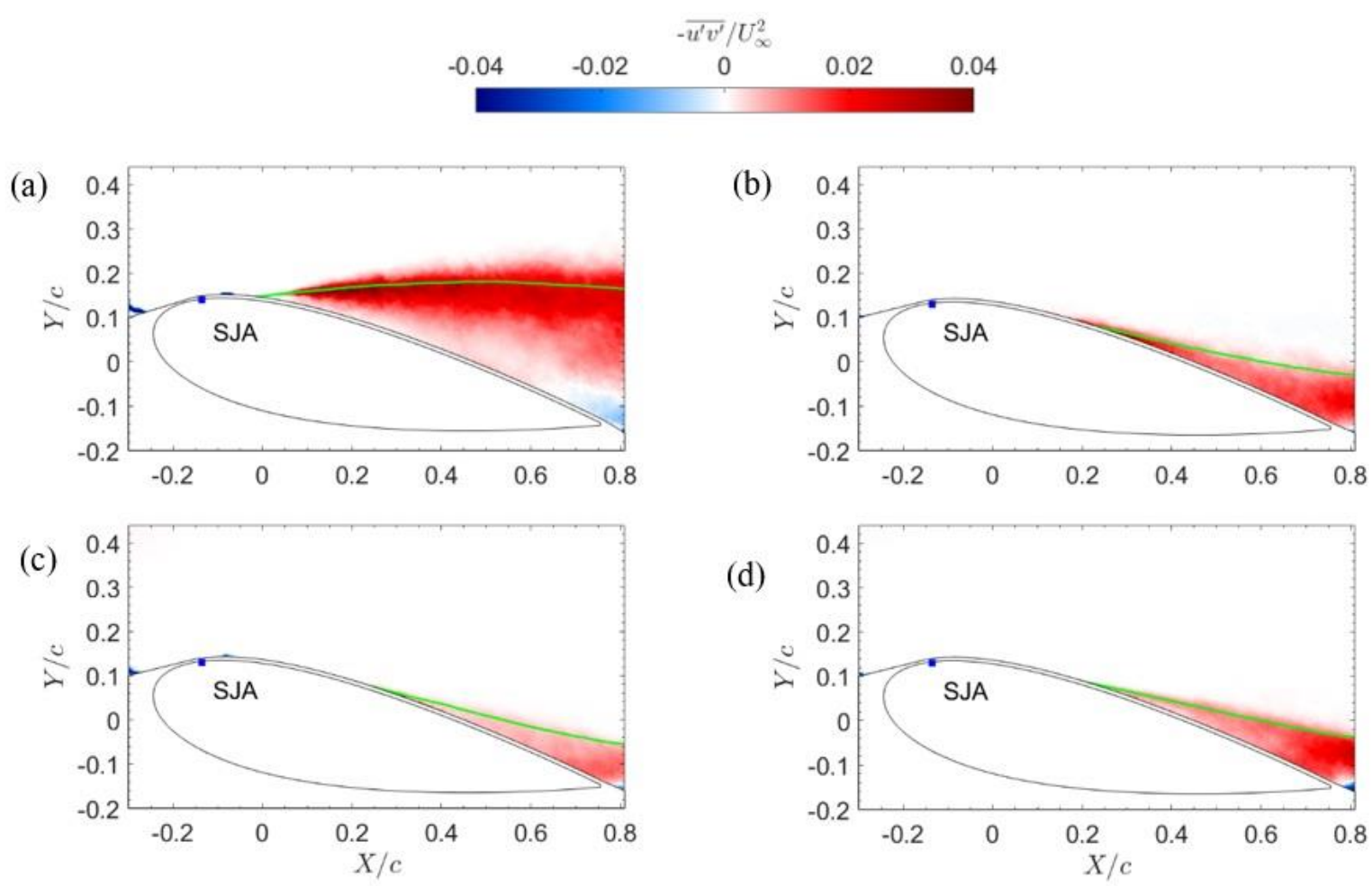

2 FIG. 5. Contours of the Reynolds shear stress for (a) Baseline, (b) F1, (c) F14 and (d) F58.

3 A quantitative comparison of the Reynolds stresses between the five test cases are presented in Fig. 6, where

4 the distributions of the maximum normalized Reynolds stresses have been plotted for each streamwise

5 location. The normal stresses demonstrate strong anisotropy $\left(\overline{u^{\prime} u^{\prime}}>\overline{v^{\prime} v^{\prime}}\right)$ of the turbulence field in each

6 test case. The peaks of the normal stresses provide a measure of the vortex formation length $\left(L_{v}\right)^{43,44}$. Here,

7 the vortex formation length for the baseline flow is defined at the streamwise distance from the separation

8 point to the location of the peak streamwise Reynolds normal stress. For the controlled cases, the vortex

9 formation length is measured from the streamwise location of the onset of the attached flow $(\mathrm{X} / c=0.2)$.

10 The vortex formation length is $L_{v} / c=0.2$ for the baseline flow, but dramatically reduces to $L_{v} / c=0.1$ for F1

11 and gradually diminishes with increasing actuation frequency. This demonstrates the damping effect of the

12 SJA actuation on the separation and vortex roll-up as the actuation frequency increases. The profiles also

13 show a significant decrease in Reynolds stresses from the baseline flow to the SJA flow. Differences

14 between the four different frequencies can also be observed where F14 has the lowest maximum Reynolds

15 stress profiles because actuation at the shear layer frequency completely suppresses large-scale vortex

16 shedding. An increase in the control of turbulent wake and decrease in Reynolds stress values can be

17 observed with higher reduced frequencies until F14. However, F58 showed an increase in Reynolds stress

18 values because the high frequency excitation is not as effective as F14 in suppressing the large-scale vortex

19 shedding and flow separation (see Fig. 2). At F1, there is a spike in the shear stresses at the SJA location.

20 This is greater than found at F2, F14 or F58. However, for F58, the maximum value of Reynolds stress is 
greater than that found for the other actuation frequencies. The control effectiveness is not as good as found

2 at the lower actuation frequencies. Fig. $6 \mathrm{~d}$ is the distributions of the Townsend structure parameter, showing

3 the $-\overline{u^{\prime} v^{\prime}} / 2 k$ (where $k=0.5\left(\overline{u^{\prime} u^{\prime}}+\overline{v^{\prime} v^{\prime}}\right.$ ) is the turbulent kinetic energy) values reach $0.15 \pm 0.05$, which 4 agrees with the standard modelling coefficient of $C_{\mu}=\left(-\overline{u^{\prime} v^{\prime}} / k\right)^{2} \approx 0.09$ in eddy viscosity models. ${ }^{45}$

(a)
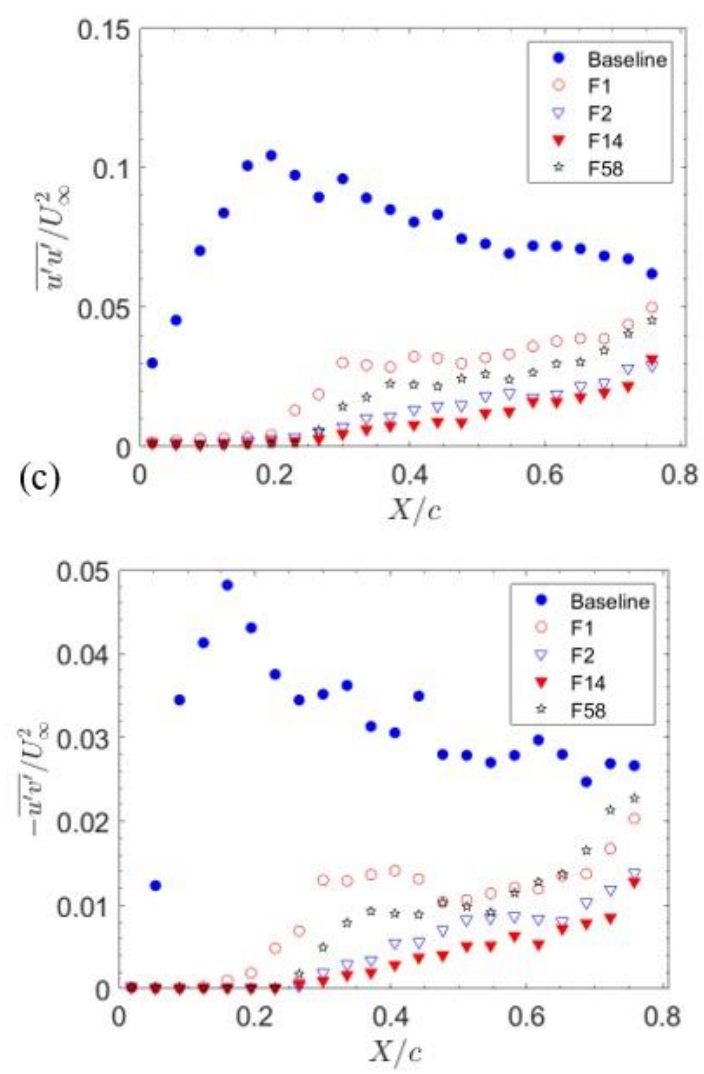

(b)
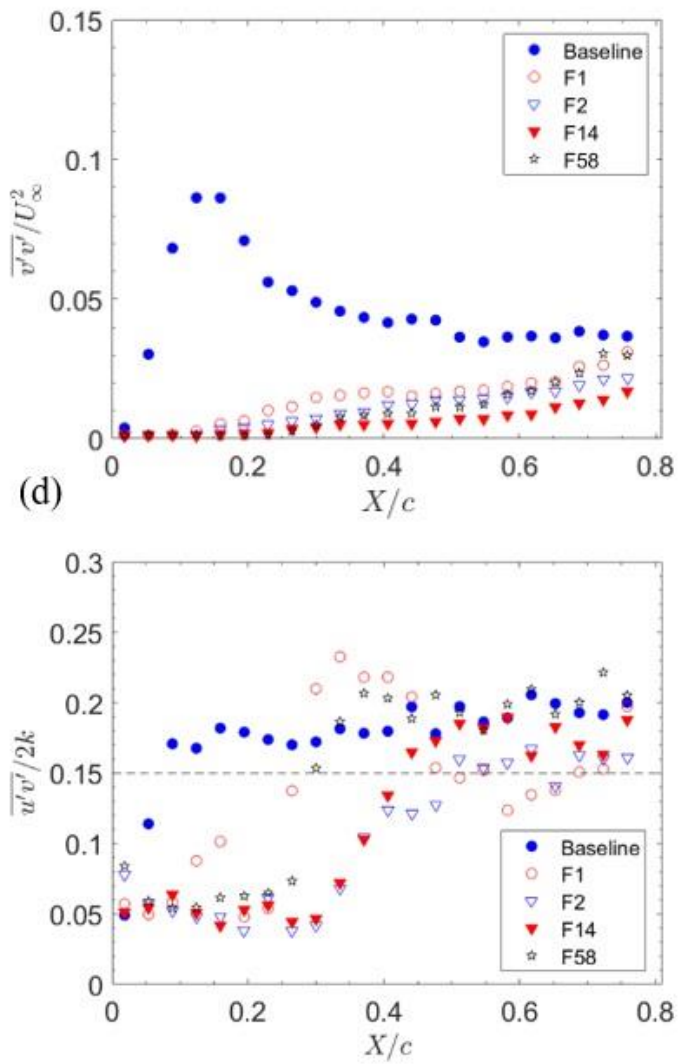

FIG. 6. Distribution of (a) normalized maximum streamwise Reynolds normal stress, (b) normalized maximum transverse Reynolds normal stress, (c) normalized maximum Reynolds shear stress, and (d) the Townsend structure parameter.

\section{Turbulent production}

Studying the effect of reduced frequency actuation on turbulence production, contours of the production term of the turbulent kinetic energy transport equation for the baseline flow, F1, F14 and F58 are presented in Fig. 7. The plots for F2 are qualitatively similar to those of F1, therefore, not shown for brevity. Here, the turbulent kinetic energy production of the 2D flow field is calculated as $P_{t k e}=-\left(\bar{u}^{2} \frac{\partial U}{\partial x}+\bar{v}^{2} \frac{\partial V}{\partial y}\right)-$ $\overline{u v}\left(\frac{\partial U}{\partial y}+\frac{\partial V}{\partial x}\right)$. The turbulent kinetic energy production was broken down into contribution from the normal stresses $P_{N}=-\left(\bar{u}^{2} \frac{\partial U}{\partial x}+\bar{v}^{2} \frac{\partial V}{\partial y}\right)$ and the shear stresses $P_{S}=-\overline{u v}\left(\frac{\partial U}{\partial y}+\frac{\partial V}{\partial x}\right) \cdot{ }^{46}$ The contours of the 
1 normalized $P_{N}$ and $P_{S}$ for the baseline flow and F14 are shown to complement the results of the total

2 production in Fig. 8. The production from the normal stresses shows a negative contour, while the

3 production from the shear stresses shows a positive contour with the normal stresses acting as an energy

4 sink, while the shear stresses are an energy source. For the baseline flow, the total magnitude of the

5 contribution of the shear stresses are an order of magnitude higher than the total magnitude of the

6 contribution of the normal stresses. This can be understood as the contribution of $-\overline{u v} \frac{\partial U}{\partial y}$, which is the

7 dominant turbulence production component in the turbulent boundary layer. A comparison between the

8 baseline flow and F14 shows that the baseline flow has a magnitude $4.1 \times$ the total contribution of the shear

9 stresses, while F14 has a magnitude $6.8 \times$ the normal stresses of the baseline flow. It is also noted that

10 production is moved from the shear layer boundary in the baseline flow to near the airfoil surface for F14.

11 The SJA actuator in F14 acts as an energy sink, convecting turbulent kinetic energy into the boundary layer.

12 The SJA draws lower momentum fluid into the cavity during the ingestion phase, bringing the higher

13 momentum fluid at the boundary layer edge to the wall, and introduces new momentum to the flow during

14 the ejection phase.
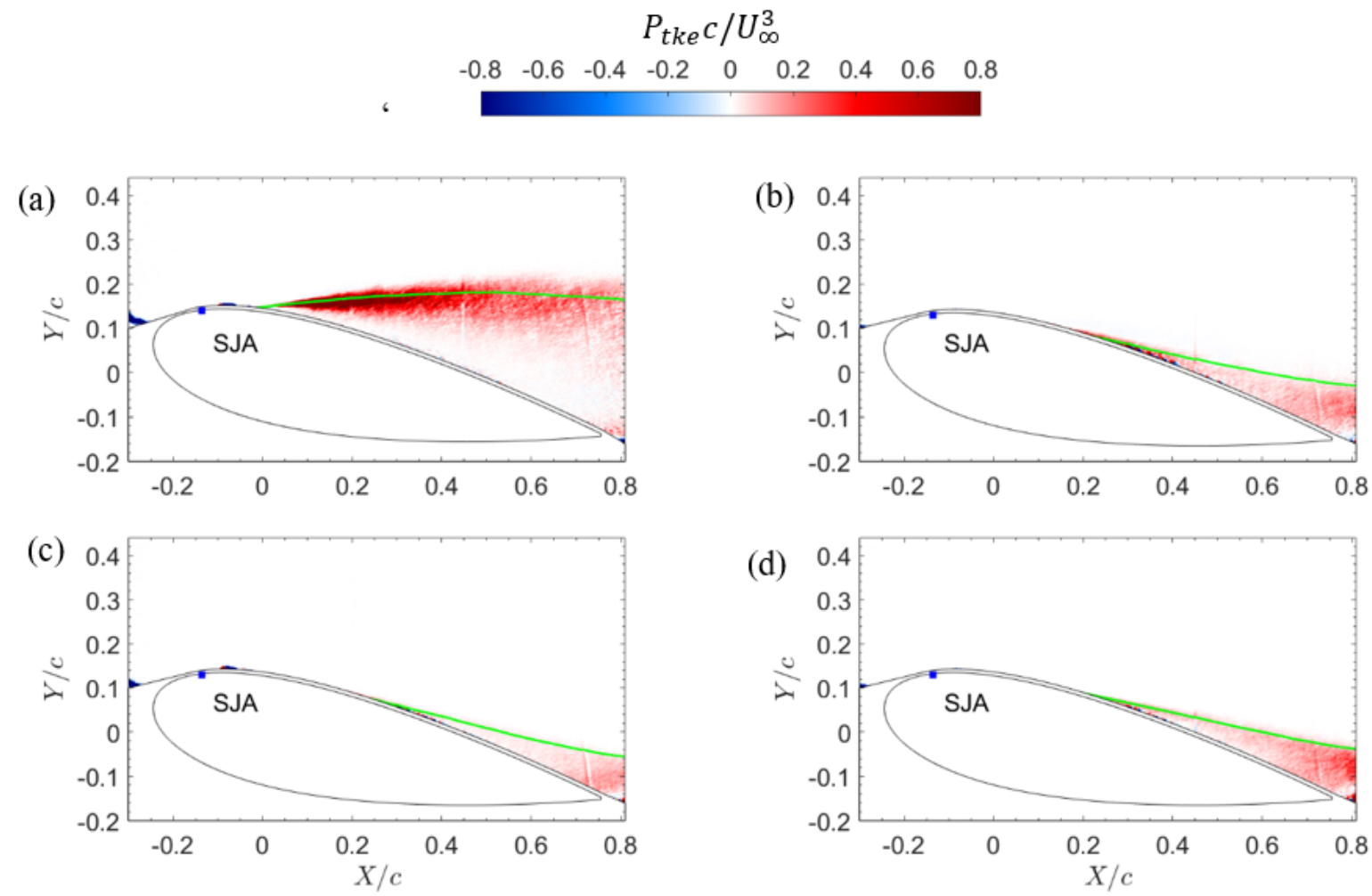

16

FIG. 7. Contours of the production of turbulent kinetic energy for (a) Baseline, (b) F1, (c) F14, and (d) F58. 

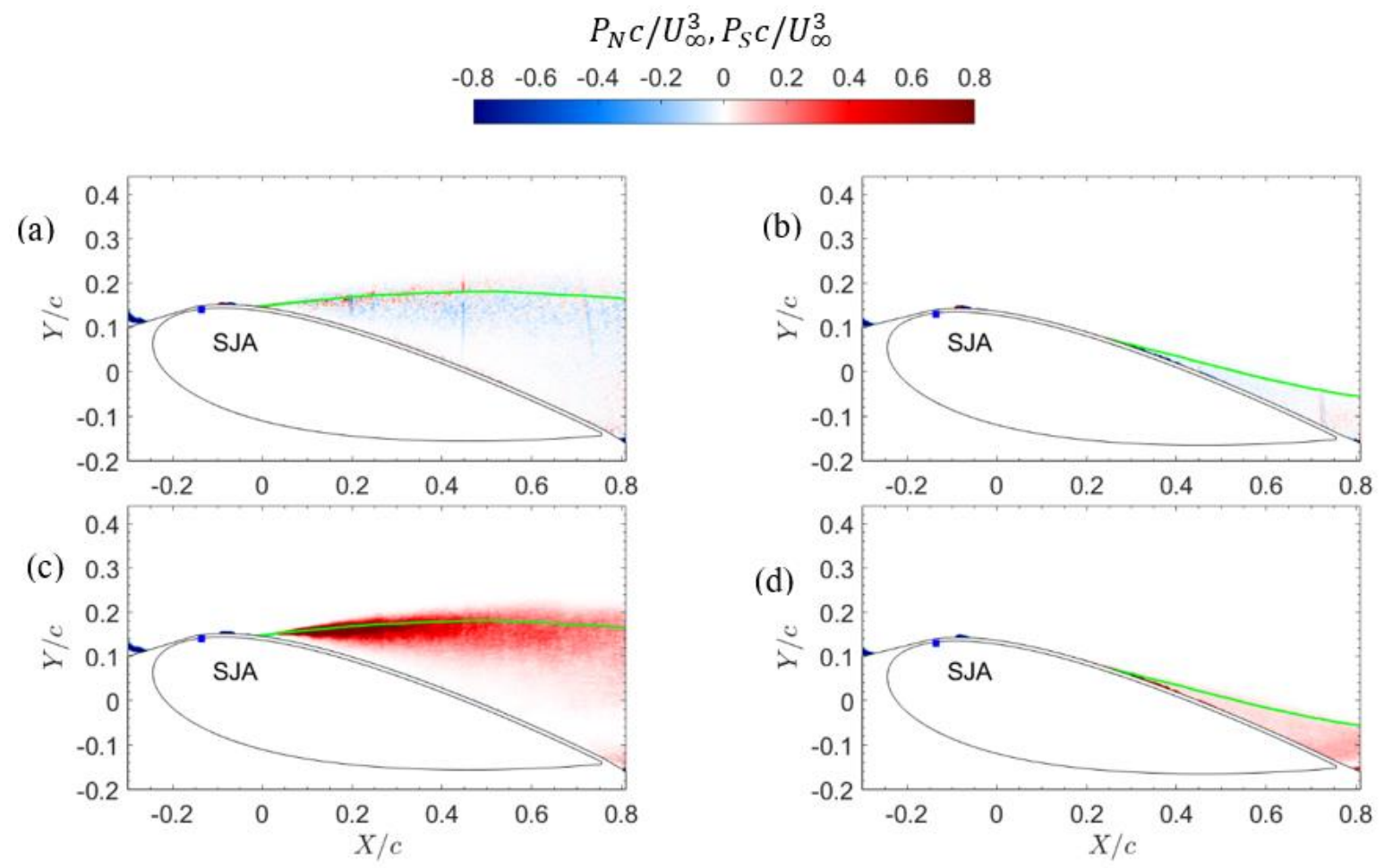

FIG. 8. Contours of the contributions of the normal stresses, $P_{N}(a, b)$ shear stresses, $P_{S}(c, d)$ to total production of turbulent kinetic energy for the baseline flow (a, c) and F14 (b, d).

\section{D. Instantaneous flow field}

5 Figure 9 shows the contours of the instantaneous vorticity and a Galilean decomposition of instantaneous

6 velocity vectors superimposed on it based on a uniform convection velocity of $U_{c}=0.9 U_{\infty}$ for the baseline

7 flow and the two extreme controlled cases (F1 and F58). For the baseline flow, a vortex roll-up phenomena

8 can be observed between $X / c \in[0,0.2]$ coinciding with the region of increasing Reynolds stresses in Fig.

9 6(a)-(c). Previous studies have found that the vortex roll-up plays a key role in transition to turbulence. ${ }^{32}$

10 In the case of F1 and F58, the vortex roll-up is completely suppressed, leading to a low level of turbulence

11 (Fig. 4-Fig. 6) and reduced vorticity on the surface of the airfoil. The damping effects of the SJA on the

12 vorticity increases with increasing forcing frequency. 

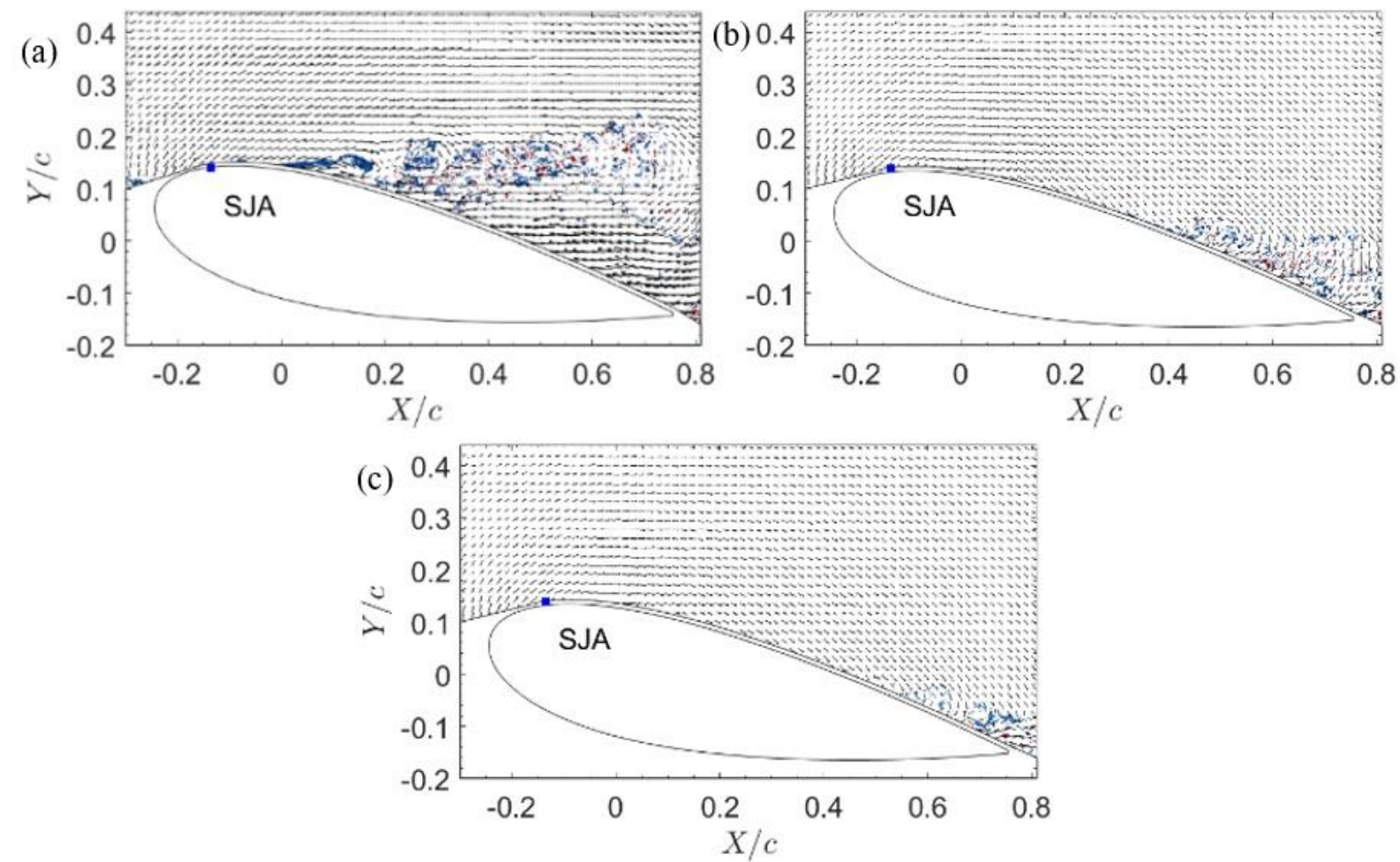

2 FIG. 9. Galilean decomposition of the instantaneous velocity field superimposed on contours of 3 instantaneous vorticity for (a) Baseline, (b) F1, and (c) F58. The contour level is $\pm 50 U_{\infty} / c$ and the 4 convection velocity applied is $U_{c}=0.9 U_{\infty}$. Red contours are positive and blue contours are negative.

\section{E. Proper orthogonal decomposition}

6 Proper Orthogonal Decomposition (POD) can provide insight into the flow organization and be useful to study how the various actuation frequencies affect the energetic flow structures. POD aims to find the spatially orthogonal structures or patterns embedded in the flow. The fluctuating velocity component $u(x, t)$ can be defined as the sum of eigenmodes as:

$$
u(x, t)=\sum_{i=1}^{N} a_{i}(t) \phi_{i}(x)
$$
where $\phi_{i}(x)$ are the eigenfunctions at the $\mathrm{i}^{\text {th }}$ mode, and $a_{i}(t)$ is the coefficients corresponding to the $\mathrm{i}^{\text {th }}$ mode. To find the spatial orthogonal eigenfunctions, POD was used. The $u$ ' and $v$ ' velocity vectors of the flow formed matrix $A$ where the streamwise and transverse velocity perturbation values of each time step were put into a single column vector using 500 timesteps. Then a POD was performed on $A$ as:

$$
A=W \sum C^{*}
$$

where $W$ is a $N \times N$ orthogonal matrix representing spatial orthogonal modes, and $C$ is a $M \times M$ matrix representing the the temporal modes, and $\sum$ is a $N \times M$ matrix, in which is diagonal component of $\sum^{2}$ 
1 represent the turbulent kinetic energy. $\sum$ is ranked in terms of its magnitude, and its corresponding spatial

2 and the temporal modes are also ranked based on its energy. The lower-order modes with most of the

3 turbulent kinetic energy are often associated with the large-scale energetic structures while the higher-order

4 modes are associated with the smaller-scale structures.

5 Figure 10 shows the fractional and cumulative energy percentage at each mode for the baseline flow and 6 the four forcing frequencies. The fractional energy distributions of the five test cases show a similar 7 tendency with the first few modes having significantly higher energy and decaying asymptotically beyond 8 mode 50. The impact of the SJA on the energy content of the structures is evident in the first 7 modes, 9 beyond which there is limited difference between the flows considered here. This indicates that the ejection 10 of the vortex pair from the SJA has stronger influence on the large-scale energetic structures than the 11 smaller-scale structures. For the first 7 modes, F1 has the highest energy content in the modes, while F14 12 shows the lowest energy distribution among the various test cases. This would imply that actuation at the 13 shear layer frequency (F14) is most effective in suppressing the large-scale structures associated with vortex 14 shedding, but actuation at the wake frequency (F1) augment the energy content of these structures. Figure 1510 (a) and (b) also suggests that further increase in frequency from F14 to F58 regenerates the energy of 16 these large-scale structures. Figure 10(c) demonstrates that, after the first 7 modes, the baseline flow attains 17 the highest energy content up to $\sim 250$ modes, corresponding to a cumulative energy of $70 \%$, and then the 18 cumulative energy percentage decreased from the baseline case to F1, F2, and F14, increasing back at F58. 19 It can be thought that the range of scales, which signifies the complexity of the overall flow structure, 20 increased with higher frequency values until F14, and possibly the deconstruction of this complex structure 21 took place at F58. 
(a)

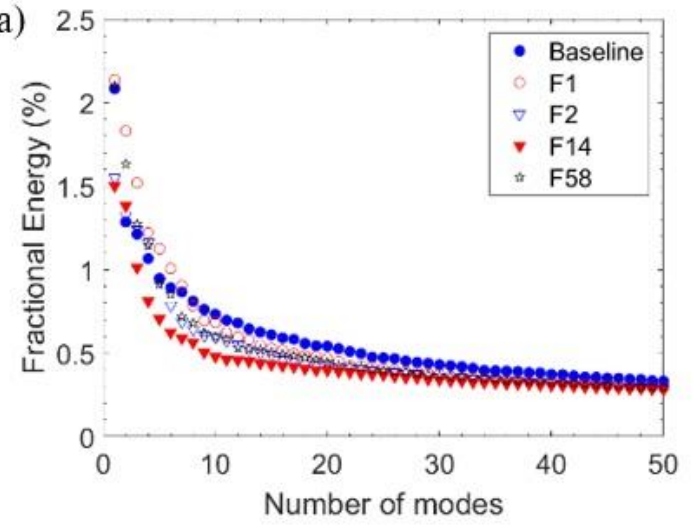

(b)

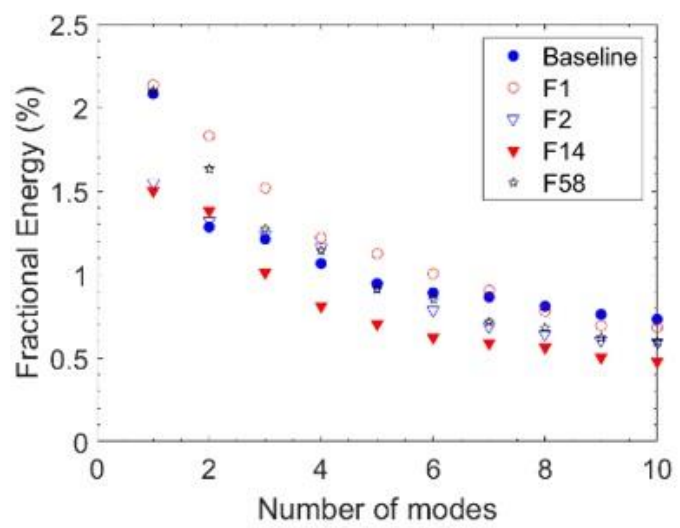

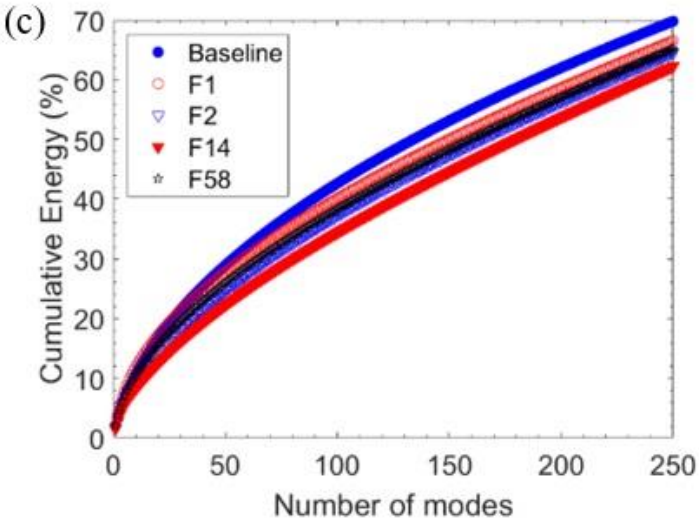

2 FIG. 10. Fractional energy distributions for (a) first 50 modes and (b) first 10 modes, and (c) cumulative 3 energy distributions for Baseline, F1, F2, F14 and F58.

4 Figures 11 and 12 present POD modes 1-3 for the baseline flow, F1, F14, and F58. The contours in Fig. 11 5 are the turbulent kinetic energy modes for the streamwise flow, and the contours in Fig. 12 are the turbulent 6 kinetic energy modes for the transverse flow. Figure 11a-c shows the first three modes for the baseline flow, 7 demonstrating a turbulent boundary layer for the mode 1, and a large vortex related to the vortex shedding 8 phenomena in modes $2 \& 3$. For F1 (figs 11(d)-(f)), the first three modes reveal an organized structure of 9 alternating vortices attached to the airfoil surface and extending into the freestream. These organized structures alternately entrain freestream fluid towards the airfoil surface and eject fluid away from the

11 surface towards the edge of the shear layer, which may partially explain the higher energy content in these 12 modes for F1 than the other test cases (see Fig. 10(b)). Figures 11g-i and 12g-i show the streamwise and 13 transverse modes for modes 1-3 for F14, and Fig. 11j-1 and Fig. 12j-1 show the corresponding plots for F58. 14 While a similar flow structure is observed for modes 1-3 for F14 and F58, the development of the vortices 15 is closer to the trailing edge for F14, indicating a higher suppression for F14 as was seen with the attached 16 flow which had a reduced wake width. F14 showed the most effective flow control and at a higher 17 frequency, F58, the effectiveness of the control decreased. Figures 13 and 14 compare modes 4-6 in its 18 streamwise and transverse direction for the four different flow conditions. A more definitive vortex 
1 shedding phenomena can be observed in mode $4 \& 5$ for the baseline flow, and a slight upward movement

2 in F58 in comparison to F14 can be observed. Additionally, the modes extending out of the boundary layer

3 can be observed for F1 for all the modes, indicating a partial suppression of the flow.
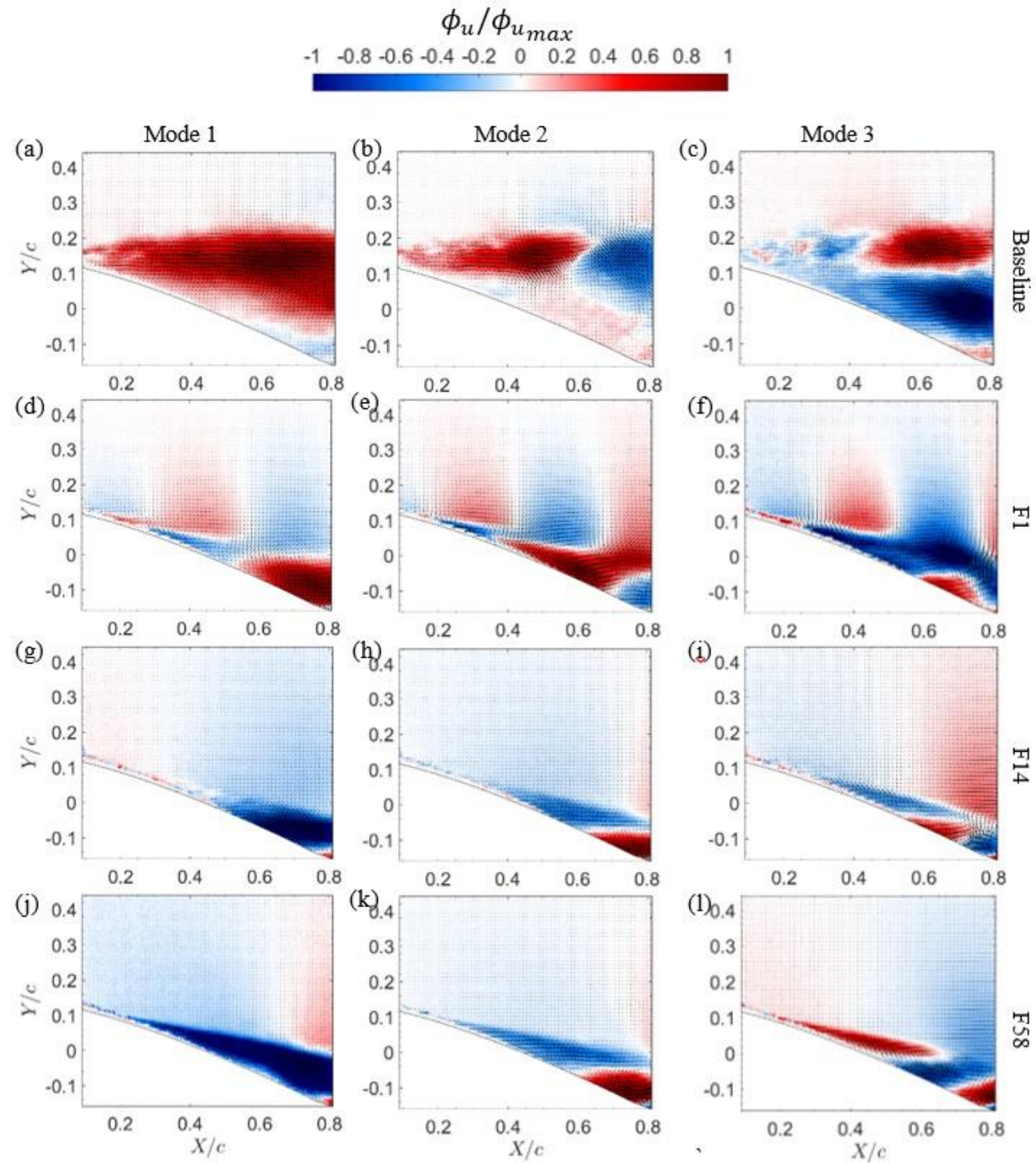

5

FIG. 11. Contours of $\phi_{\mathrm{u}}$ of POD mode 1,2 , and 3 with superimposed vectors of $\phi_{\mathrm{u}}$ and $\phi_{\mathrm{v}}$ for baseline (a 6 c), F1(d-f), F14(g-i) and F58(j-1) 

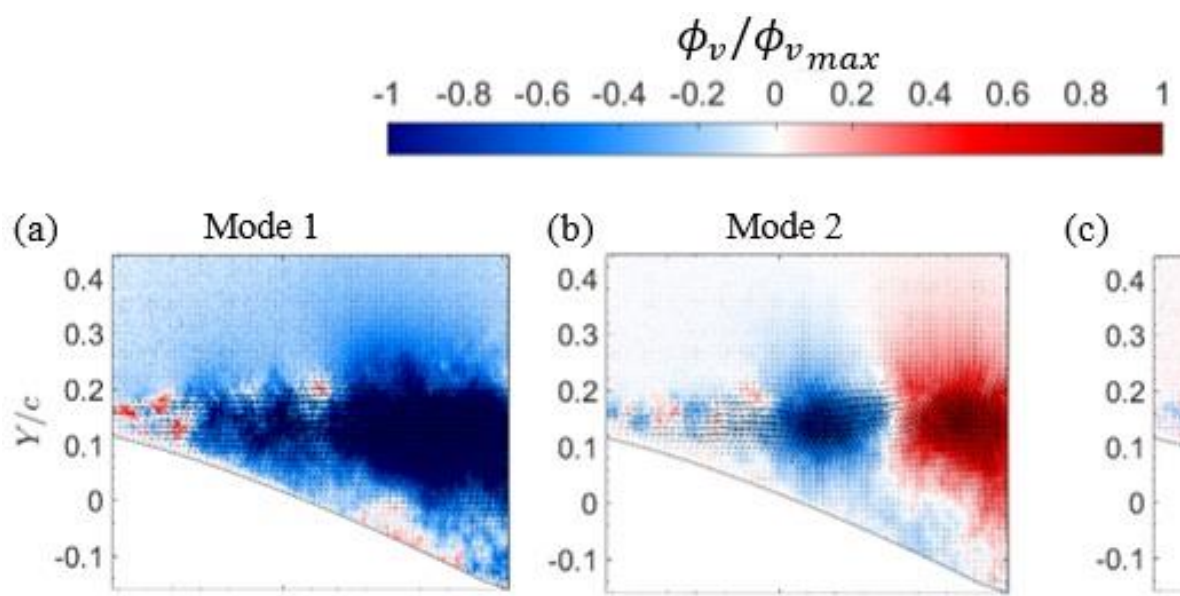

(b) Mode 2
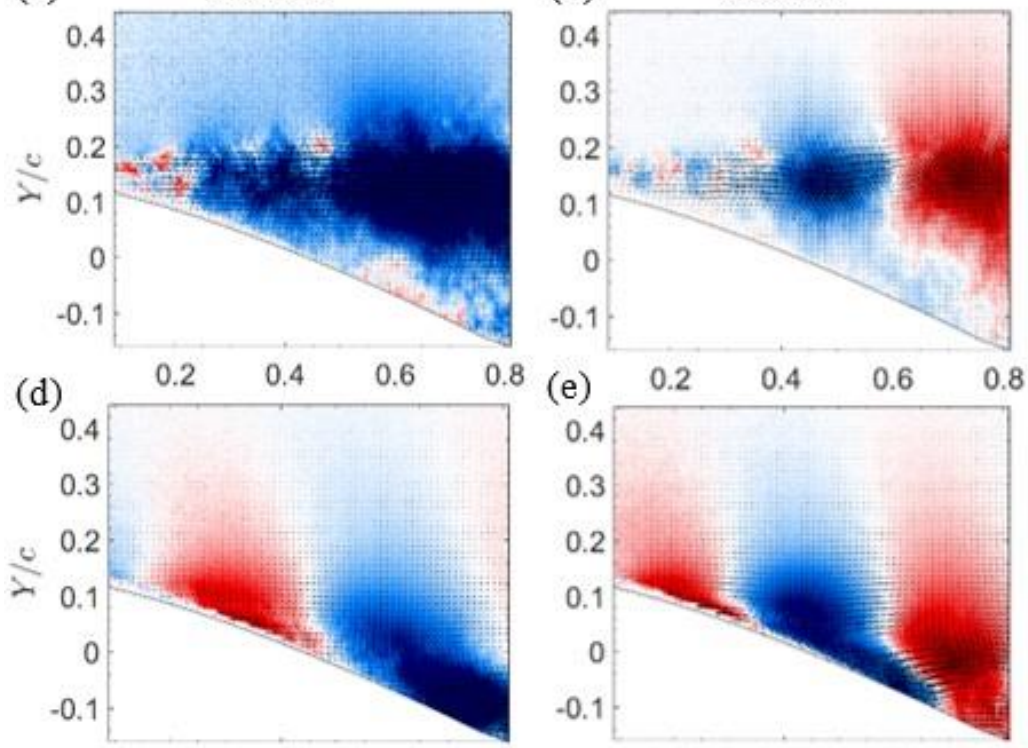

(c) Mode 3
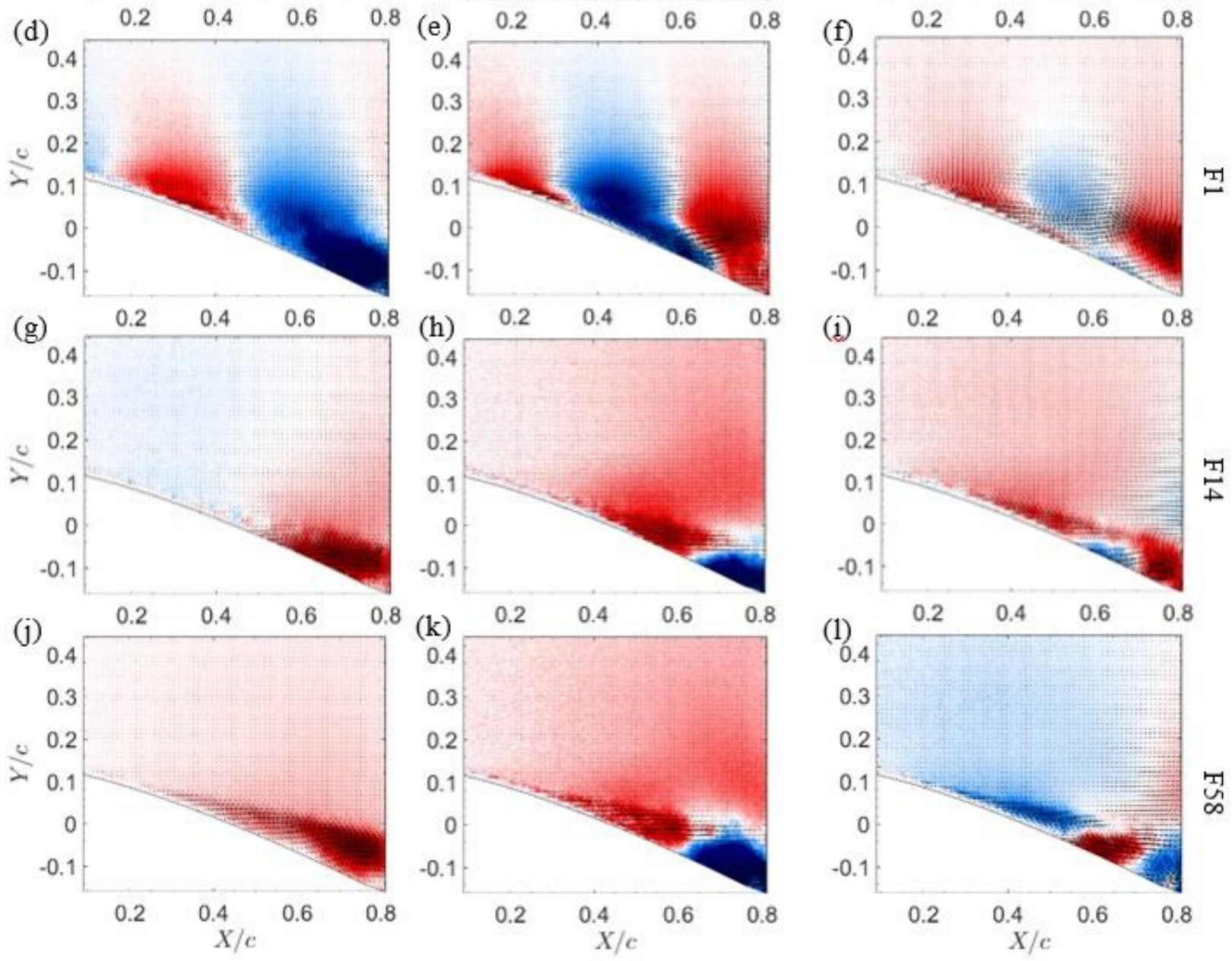

2 FIG. 12. Contours of $\phi_{\mathrm{v}}$ of POD mode 1, 2, and 3 with superimposed vectors of $\phi_{\mathrm{u}}$ and $\phi_{\mathrm{v}}$ for baseline (a 3 c), F1(d-f), F14(g-i) and F58(j-1) 

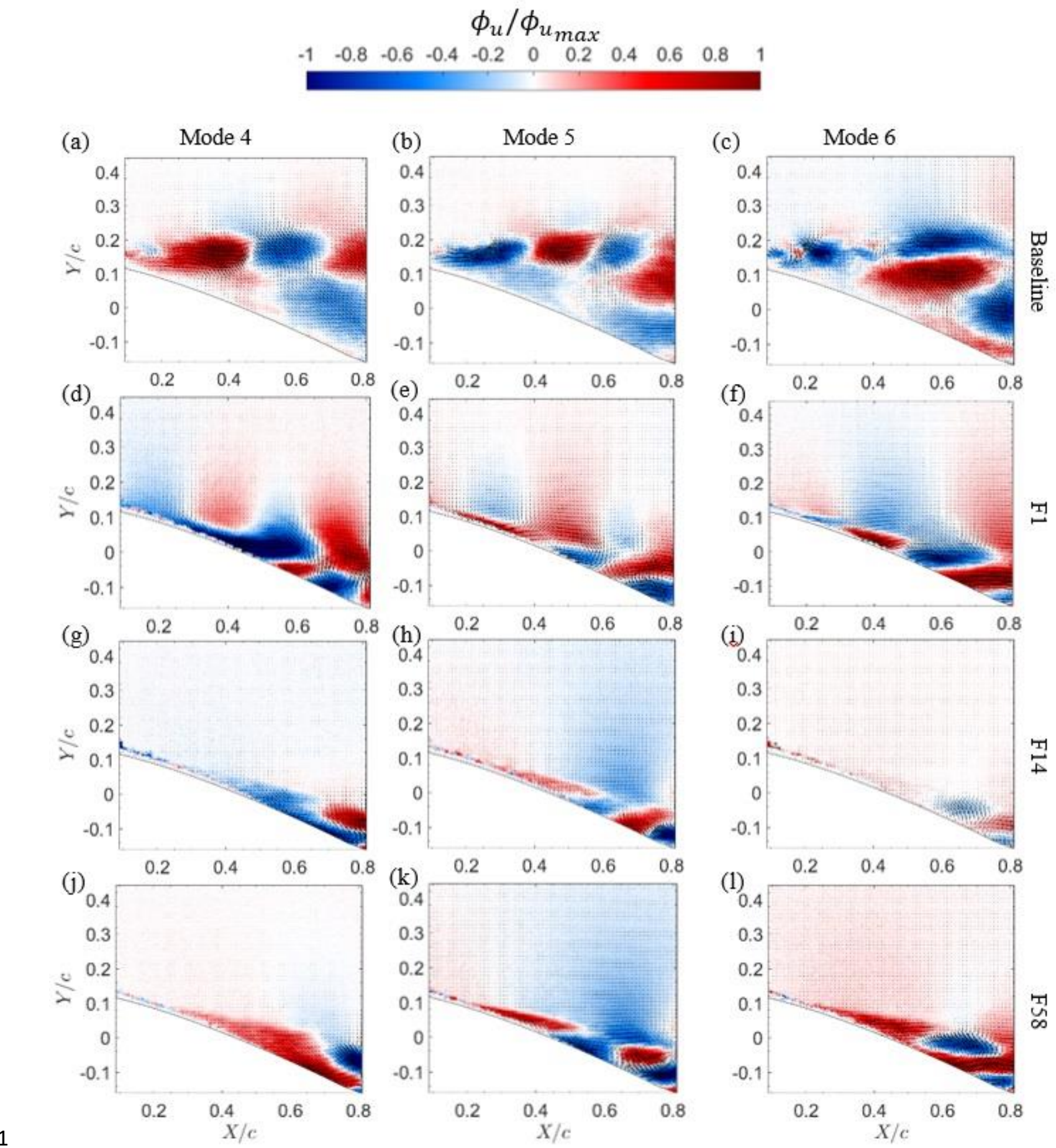

2 FIG. 13. Contours of $\phi_{\mathrm{u}}$ of POD mode 4, 5, and 6 with superimposed vectors of $\phi_{\mathrm{u}}$ and $\phi_{\mathrm{v}}$ for baseline (a 3 c), F1(d-f), F14(g-i) and F58(j-1) 


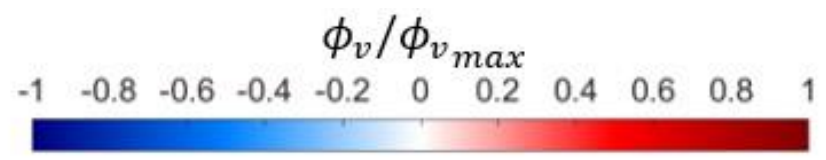

(a)

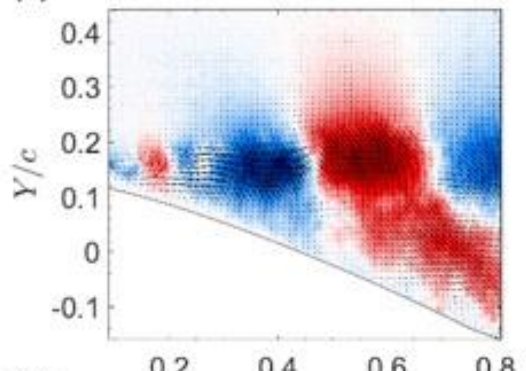

(d)
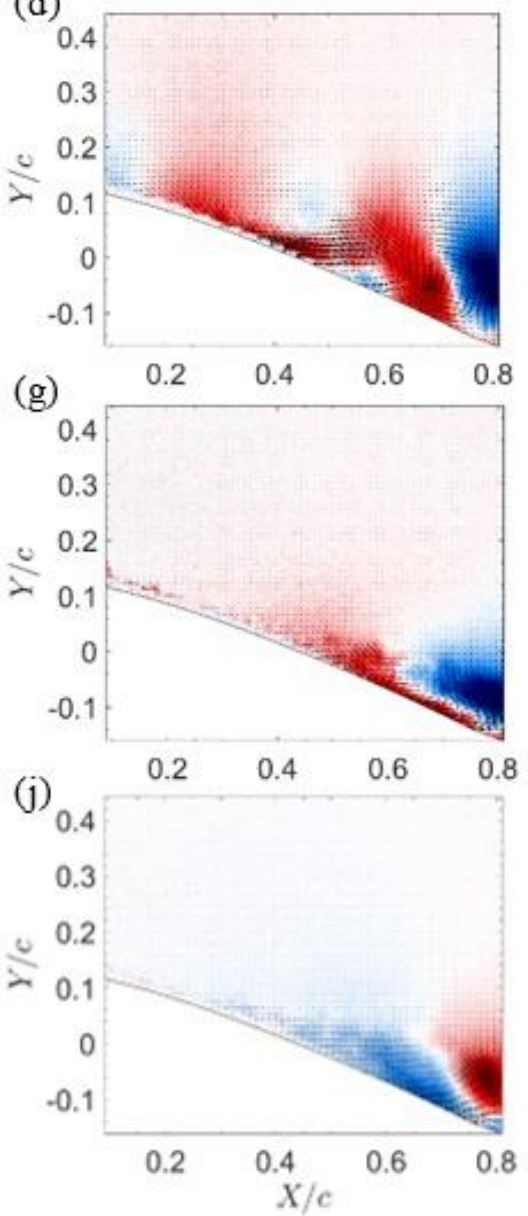

(b)

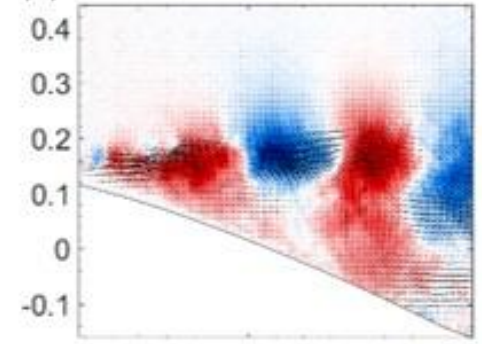

(e)

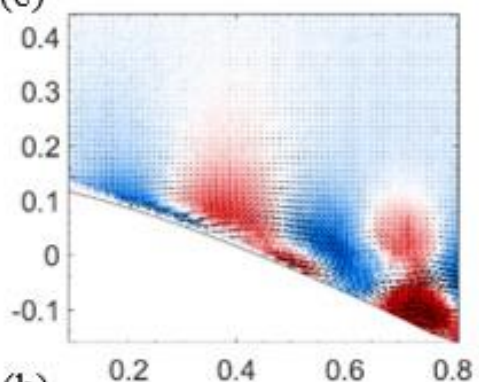

(h)
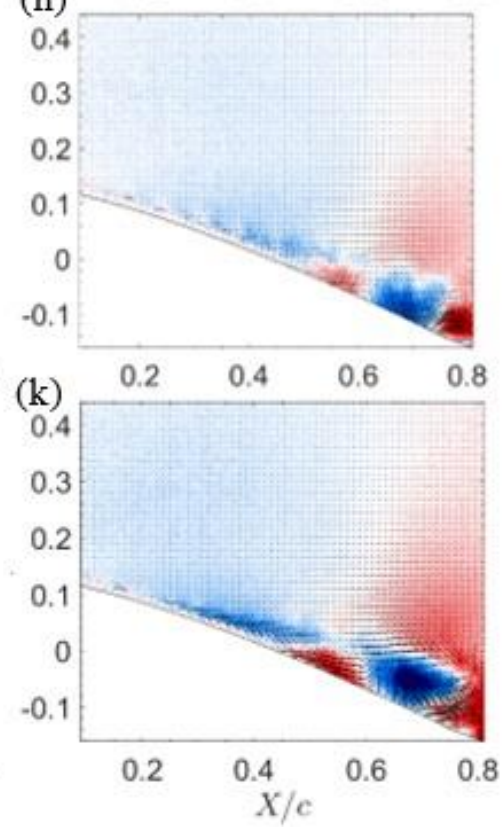

(c)

Mode 6

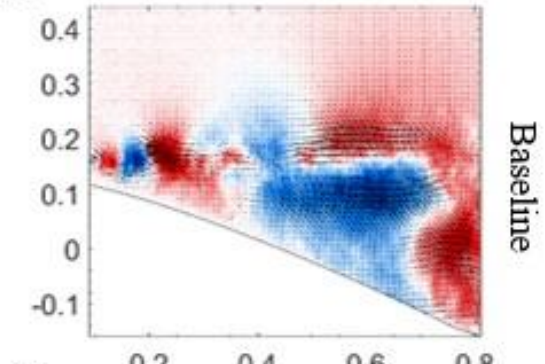

(f)
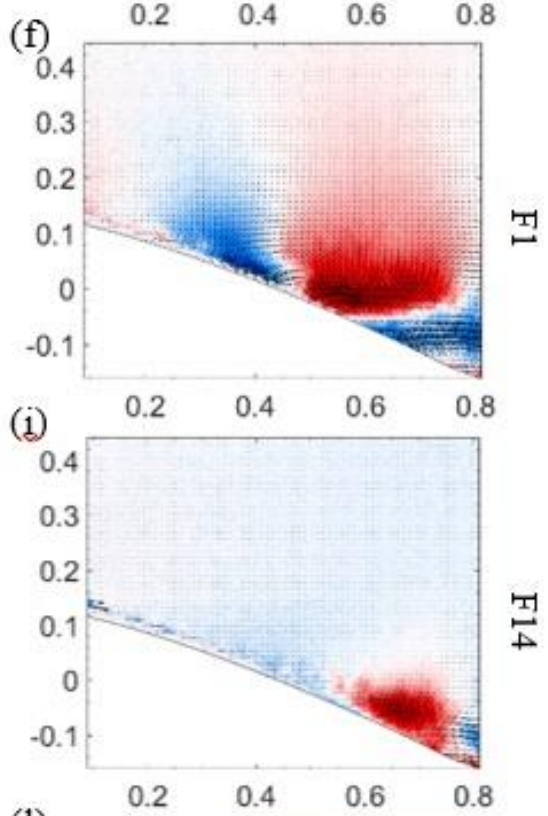

(1)

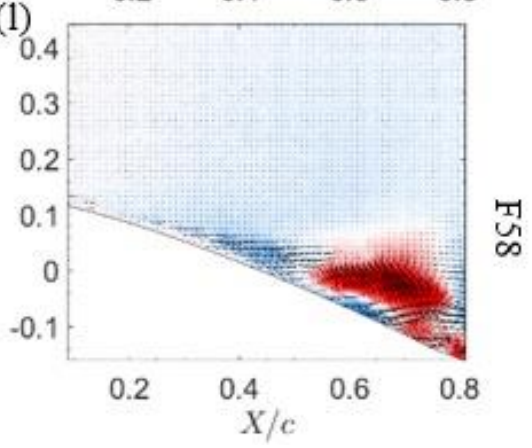

\section{IV. CONCLUSION}

5 The impact of synthetic jet actuation on aerodynamic control at wake frequencies (F1 and F2), shear layer

6 frequency (F14) and high frequency (F58) was investigated. The turbulent kinetic energy production

7 showed that the SJA acts as a turbulent kinetic energy sink. Turbulence production was highest for the 
1 baseline flow, followed by F1, a decrease in turbulent energy until F14, and showing an increase back again

2 in F58, demonstrating the effectiveness range of the controlled frequencies in turbulent wake suppression.

3 From POD, the coherent structures showed that low controlled frequencies were associated with large scale

4 ejections that propagated to the outer boundary of the shear layer. Increasing the frequency showed smaller

5 scale momentum input confined to the shear layer. Increasing the frequency was ineffective as it does not

6 generate sufficient momentum per stroke to affect the shear layer. This suggests F1 and F14 are effective

7 not just for their average input but the momentum/cycle.

\section{ACKNOWLEDGEMENTS}

9 The authors would like to acknowledge support from the Natural Sciences and Engineering Research 10 Council of Canada and CMC Microsystems. Computations were performed at the SciNet HPC Consortium.

11 SciNet is funded by the Canada Foundation for Innovation under the auspices of Compute Canada, the

12 Government of Ontario, Ontario Research Fund - Research Excellence, and the University of Toronto.

13 SOSCIP is funded by the Federal Economic Development Agency of Southern Ontario, IBM Canada, Ltd.,

14 Ontario Centres of Excellence, Mitacs, and 14 academic member institutions. The authors thank Dr. Mark

15 Feero for the data.

\section{REFERENCES}

$17{ }^{1}$ P.B.S. Lissaman, Annu. Rev. Fluid Mech. 15, 223 (1983).

$18 \quad{ }^{2}$ H. Hu and Z. Yang, J. Fluids Eng. Trans. ASME 130, 0511011 (2008).

$19 \quad{ }^{3}$ M. Gad-el-Hak, J. Aircr. 38, 419 (2001).

$20{ }^{4}$ M.A. Feero, S.D. Goodfellow, P. Lavoie, and P.E. Sullivan, AIAA J. 53, 2005 (2015).

$21{ }^{5}$ H. Shan, L. Jiang, C. Liu, M. Love, and B. Maines, Comput. Fluids 37, 975 (2008).

$22{ }^{6}$ T. Moghaddam and N.B. Neishabouri, IOP Conf. Ser. Mater. Sci. Eng. 248, 0 (2017).

$23{ }^{7}$ S. Siva Viknesh and K. Poddar, Phys. Fluids 33, (2021).

$24{ }^{8}$ Z. Li, D. Zhang, Y. Liu, C. Wu, and N. Gao, Phys. Fluids 32, (2020).

$25 \quad{ }^{9}$ D. Greenblatt and I.J. Wygnanski, Prog. Aerosp. Sci. 36, 487 (2000).

$26{ }^{10}$ S.D. Goodfellow, S. Yarusevych, and P.E. Sullivan, AIAA J. 51, 623 (2013). 
${ }^{11}$ M.A. Feero, P. Lavoie, and P.E. Sullivan, Exp. Fluids 58, 99 (2017).

$2 \quad{ }^{12}$ D. Gao, H. Meng, Y. Huang, G. Chen, and W.L. Chen, Phys. Fluids 33, (2021).

$3 \quad{ }^{13}$ L. Xia, Y. Hua, and J.G. Zheng, Phys. Fluids 33, (2021).

$4 \quad{ }^{14}$ A. Seifert, T. Bachar, D. Koss, M. Shepshelovich, and I. Wygnanski, AIAA J. 31, 2052 (1993).

$5 \quad{ }^{15}$ A. Glezer and M. Amitay, Annu. Rev. Fluid Mech. 34, 503 (2002).

$6 \quad{ }^{16}$ B.L. Smith and A. Glezer, Phys. Fluids 10, 2281 (1998).

$7 \quad{ }^{17}$ F.-B. Hsiao, C.-F. Liu, and J.-Y. Shyu, AIAA J. 28, 1440 (1990).

$8{ }^{18}$ J.M. Wu, X.Y. Lu, A.G. Denny, M. Fan, and J.Z. Wu, 4th Shear Flow Control Conf. 371, 21 (1997).

$9 \quad{ }^{19}$ M. Amitay, D.R. Smith, V. Kibens, D.E. Parekh, and A. Glezer, AIAA J. 39, 361 (2001).

$10{ }^{20}$ M. Amitay and A. Glezer, Int. J. Heat Fluid Flow 23, 690 (2002).

$11{ }^{21}$ J.L. Gilarranz, L.W. Traub, and O.K. Rediniotis, J. Fluids Eng. Trans. ASME 127, 377 (2005).

$12{ }^{22}$ E. Montazer, M. Mirzaei, E. Salami, T.A. Ward, F.I. Romli, and S.N. Kazi, IOP Conf. Ser. Mater. Sci.

13 Eng. 152, (2016).

$14 \quad{ }^{23}$ D. You and P. Moin, J. Fluids Struct. 24, 1349 (2008).

$15 \quad{ }^{24}$ M. Moshfeghi and N. Hur, J. Mech. Sci. Technol. 31, 1233 (2017).

$16 \quad{ }^{25}$ W. Zhang and R. Samtaney, Phys. Fluids 27, 55101 (2015).

$17{ }^{26}$ A. Seifert and L.T.G. Packt, 37th Aerosp. Sci. Meet. Exhib. (1999).

$18 \quad{ }^{27}$ R. Raju, R. Mittal, and L. Cattafesta, AIAA J. 46, 3103 (2008).

$19{ }^{28}$ R. Kotapati, R. Mittal, and L. Cattafesta, in 44th AIAA Aerosp. Sci. Meet. Exhib. (2006), p. 320.

$20 \quad{ }^{29}$ N.A. Buchmann, C. Atkinson, and J. Soria, Exp. Fluids 54, (2013). 
$1 \quad{ }^{30}$ A. Glezer, M. Amitay, and A. Honohan, AIAA J. 43, 1501 (2005).

$2 \quad{ }^{31}$ R. Duvigneau and M. Visonneau, Comput. Fluids 35, 624 (2006).

$3 \quad{ }^{32}$ S. Yarusevych, P.E. Sullivan, and J.G. Kawall, Phys. Fluids 18, 44101 (2006).

$4 \quad{ }^{33}$ S. Yarusevych, P.E. Sullivan, and J.G. Kawall, J. Fluid Mech. 632, 245 (2009).

$5 \quad{ }^{34}$ Y. Tian, L.N. Cattafesta, and R. Mittal, in 44th AIAA Aerosp. Sci. Meet. Exhib. (Reno, NV, United

$6 \quad$ States, 2006), pp. 2006-1401.

$7 \quad{ }^{35}$ A. Prasad and C.H.K. Williamson, J. Fluid Mech. 333, 375 (1997).

$8{ }^{36}$ T. Wei and C.R. Smith, J. Fluid Mech. 169, 513 (1986).

$9 \quad{ }^{37}$ M.S. Bloor, J. Fluid Mech. 19, 290 (1964).

$10{ }^{38}$ M. Amitay and A. Glezer, AIAA J. 40, 209 (2002).

$11{ }^{39}$ M.A. Feero, Investigation of Synthetic Jet Flow Control Parameters for the Mitigation of Laminar 12 Boundary Layer Separation, University of Toronto (Canada), 2018.

$13 \quad{ }^{40}$ A.K. Prasad, R.J. Adrian, C.C. Landreth, and P.W. Offutt, Exp. Fluids 1992132 13, 105 (1992).

$14 \quad{ }^{41}$ D.J. Forliti, P.J. Strykowski, and K. Debatin, Exp. Fluids 2000285 28, 436 (2000).

$15 \quad{ }^{42}$ A. Sciacchitano and F. Scarano, Meas. Sci. Technol. 25, 084009 (2014).

$16 \quad{ }^{43}$ J.H. Gerrard, J. Fluid Mech. 25, 401 (1966).

$17 \quad{ }^{44}$ B. Cantwell and D. Coles, J. Fluid Mech. 136, 321 (1983).

$18{ }^{45}$ N. Kang, E.E. Essel, V. Roussinova, and R. Balachandar, Phys. Rev. Fluids 6, 1 (2021).

$19 \quad{ }^{46}$ E.E. Essel, A. Nematollahi, E.W. Thacher, and M.F. Tachie, J. Turbul. 16, 872 (2015).

20 
